\title{
3-D Free Vibration Analysis of Doubly-Curved Shells
}

\begin{abstract}
The vibration analysis is presented for determining the natural frequencies and mode shapes of a class of doubly-curved shells with different boundary conditions, which can be considered to be a panel taking from the hollow torus with annular cross-section. The small strain, three-dimensional (3-D), linear elasticity theory is used to describe the governing equations of the problem, which is associated with the toroidal coordinate system $(r, \theta, \varphi)$ composed of the usual polar coordinates $(r, \theta)$ originating at sectorial cross-section center and an angle coordinate $\varphi$ originating at the toroidal center. The Chebyshev-Ritz method is used to derive the eigenvalue equation: each displacement is taken as the triplicate product of the Chebyshev polynomials in $r, \theta$ and $\varphi$ directions, multiplied by a boundary function along with a set of generalized coefficients, thus yielding upper bound values of natural frequencies. As the degree of the Chebyshev polynomials increases, frequencies converge monotonically to the exact values. The accuracy is demonstrated by convergence and comparison studies. The effects of thickness ratio, radius ratio, angle in $\varphi$ direction, initial angle and subtended angle in $\theta$ direction on natural frequencies and mode shapes are discussed in detail.
\end{abstract}

Keywords: Three-dimensional elasticity; doubly curved shell; vibration analysis; natural frequency; Chebyshev-Ritz method 


\section{Introduction}

Shells are widely used components in engineering such as aerospace, marine, nuclear and building. It is well known that the scopes of shell study are rather extensive and the configuration of shells is very varied. Therefore, they have particular attraction for architectural designers. In most cases, a shell structure takes on both the visual function and the practical function, such as the domes in churches, stadiums and museums. Melaragno [1] summarized the shell art in building design.

Various shell theories from thin shells to thick shells were developed by introducing different assumptions for approximation, e.g. Love [2], Donnell [3], Reissner [4] and Flügge [5]. A lot of researchers studied the vibrations of shells by analytical methods and numerical methods. Chaudhuri and Kabir [6] presented the Navier-type solution for cross-ply doubly curved panels using the shallow shell theories. Reddy [7] presented the exact solution for simply supported cross-ply spherical shell panels using the modified Sanders shell theory. Furthermore, Reddy and Liu [8] presented the Navier-type solutions for spherical shells using the higher-order shear deformation theory. Biglari and Jafari [9] studied the simply supported spherical sandwich panels using a refined sandwich theory. Hosseini-Hashemi and Fadaee [10] presented the closed-form solution for free vibration of moderately thick spherical shell panels. It is known that most of the analytical solutions for shell panels were limited to simply supported boundary conditions.

For the general cases, numerical methods should be used to analyze the mechanical properties of shells, such as finite element method [11], differential quadrature method [12] and meshless method [13] etc. It should be mentioned that the Ritz method has the excellent advantage of high accuracy and small computational 
cost in vibration analysis of structure elements, which is especially suitable for the parameterizing study. Liew et al. [14] summarized the study on vibrations of shallow shells. Lim et al. [15] made a detailed study on the applicable range of shallow shell theory for single curve cylindrical panels using the two-dimensional simple polynomials as admissible functions. Quta and Leissa [16,17] studied the free vibration of shallow shells with two adjacent edges clamped and examined the effect of edge constraints on frequencies of shallow shells using the algebraic polynomials as admissible functions. Furthermore, Narita and Liessa [18] studied the vibration of completely free shallow shells with curvilinear planform. Based on the Kirchhoff-Love theory, the vibration characteristics of shells from cylindrical shells $[19,20]$ to doubly-curved shells [21-23] were analyzed. However, with the increase of shell thickness, the shear deformable effect becomes significant. In such a case, refined theories, e.g. first-order deformable theory [24] or higher-order theory [25], should be taken. Liew and Lim [26-28] made a systematic study on the vibration characteristics of doubly-curved thick shallow shells using the two-dimensional polynomials as admissible functions in the Ritz method.

It is well known that the exact elasticity theory does not reply on any hypotheses involving the kinematics of deformation. Using the three-dimensional (3-D) elasticity theory, a complete set of frequency spectrum without missing any modes could be obtained, which cannot otherwise be predicted by the approximate theories. Such an analysis not only provides the realistic results but also allows overall physical insights. Compared with the works based on various shell theories as mentioned above, those developed directly from the exact three-dimensional linear elasticity are comparatively far fewer. Leissa and Kang [29,30] studied the 3-D vibration of thick shells of revolution and Paraboloidal shells using the algebraic polynomials as admissible functions. Also Kang and Leissa [31,32] studied the 3-D vibrations of 
thick hyperboloidal shells of revolution and thick spherical shell segments with variable thickness. Young [33] studied the 3-D vibration of doubly-curved shells with arbitrarily deep in one direction. McGee and Spry [34] studied the 3-D vibration of spherical shells of revolution. Liew et al. [35] used the one- and two-dimensional orthogonal polynomials as admissible functions to study 3-D vibrations of spherical shell panels. Lim et al. [36] studied the 3-D vibration of open cylindrical panels. Liew et al. [37] verified the accuracy of the Ritz solutions through the comparison with the finite element solutions.

It is clear that 3-D Ritz solutions are referred to the eigen-value matrices with large size. The accuracy and convergence greatly depend on the admissible functions chosen. Unsuitable admissible functions could result in bad convergence and/or instable numerical computations. As is well known, the Chebyshev polynomials [38] are a set of orthogonal polynomials with a lot of excellent mathematical properties. Using such polynomials as admissible functions can speed up the convergence of results and guarantee the numerical stability in the 3-D vibration analysis of structural components [39]. Zhou and his co-workers [40-42] studied 3-D vibrations of cylinders, annular sector plates and circular plates with varying thickness by using the Chebyshev-Ritz method. Excellent convergence and high accuracy of the method have been demonstrated. For solid/hollow rings with circular or sectorial cross-section [43-45] and circularly-curved beams with circular cross-section [46], using a set of toroidal coordinate system displays the technical convenience in 3-D vibration analysis. Under the toroidal coordinates developed, all the boundaries of the problems aforementioned are described by the constant coordinate values. In the present study, this coordinate system will be used to analyze the three-dimensional vibration of a variety of doubly-curved thick shells based on the exact small strain linear elasticity theory, combining with the Chebyshev-Ritz method. 


\section{Formulation}

Firstly, we consider a hollow ring torus with annular cross-section as shown in Figure 1. The outer radius of the cross-section is $r_{1}$ and the inner radius is $r_{0}$. The toroidal radius (the distance from the center of the torus to the center of the cross-section) is $R$. A combination of the two-dimensional polar coordinates $(r, \theta)$ with the original at the center of the cross-section and the one-dimensional angle coordinate $\varphi$ with the original at the center of the torus is chosen to describe the strains and stresses. The angle $\theta$ is measured from the torus plane. Now, we take a panel from the torus in such a way that $\varphi$ is from 0 to $\varphi_{0}$ (called toroidal angle) and $\theta$ is from $\theta_{0}$ (called initial angle) to $\theta_{1}+\theta_{0}$ ( $\theta_{1}$ is called subtended angle) as shown in Figure 1 . It can be seen from Figure 1 that various shaped shell panels can be described by taking different $\theta_{0}$ and $\theta_{1}$. Three typical shell panels are given in Figure 2, in which (a) is taken from the outer part of the torus, (b) is taken from the inner part of the torus while (c) is taken from the lateral part of the torus. It is obvious that $R=0$ means spherical shell panels and $R=\infty$ means cylindrical shell panels. The three-dimensional coordinates $(r, \theta, \varphi)$ form an orthogonal set, the position vector indicated in Figure 1 defines a typical elastic point $P$ on the torus mathematically represented parametrically as

$\overrightarrow{\mathbf{P}}=[(R+r \cos \theta) \cos \varphi] \overrightarrow{\mathbf{i}}+[(R+r \cos \theta) \sin \varphi] \overrightarrow{\mathbf{j}}+(r \sin \theta) \overrightarrow{\mathbf{k}}$

The unit vectors along the Cartesian coordinates, $\left\{\begin{array}{c}\overrightarrow{\mathbf{i}} \\ \overrightarrow{\mathbf{j}} \\ \overrightarrow{\mathbf{k}}\end{array}\right\}$, are connected to those along

the toroidal coordinates, $\left\{\begin{array}{l}\overrightarrow{\mathbf{e}}_{\mathbf{r}} \\ \overrightarrow{\mathbf{e}}_{\boldsymbol{\theta}} \\ \overrightarrow{\mathbf{e}}_{\phi}\end{array}\right\}$, as follows: 


$$
\left.\left\{\begin{array}{l}
\overrightarrow{\mathbf{e}}_{\mathbf{r}} \\
\overrightarrow{\mathbf{e}}_{\boldsymbol{\theta}} \\
\overrightarrow{\mathbf{e}}_{\varphi}
\end{array}\right\}=\left[\begin{array}{ccc}
\cos \theta \cos \varphi & \cos \theta \sin \varphi & \sin \theta \\
-\sin \theta \cos \varphi & -\sin \theta \sin \varphi & \cos \theta \\
\sin \varphi & -\cos \varphi & 0
\end{array}\right]\left\{\begin{array}{c}
\overrightarrow{\mathbf{i}} \\
\overrightarrow{\mathbf{j}} \\
\overrightarrow{\mathbf{k}}
\end{array}\right\}=[J]\right\}\left\{\begin{array}{c}
\overrightarrow{\mathbf{i}} \\
\overrightarrow{\mathbf{j}} \\
\overrightarrow{\mathbf{k}}
\end{array}\right\}
$$

The determinant of the Jacobian matrix $[\mathrm{J}]$ defining a ratio of volumetric changes in Cartesian coordinates to those in toroidal coordinates, as follows:

$$
\frac{d x d y d z}{d r d \theta d \phi}=|J|=r(R+r \cos \theta)
$$

Let $u, v$ and $w$, respectively, be the displacements in the $r, \theta$ and $\varphi$ directions, the relations between three-dimensional tensor strains and displacement components in the present coordinate system are given by

$$
\begin{aligned}
& \varepsilon_{r}=\frac{\partial u}{\partial r}, \varepsilon_{\theta}=\frac{1}{r} \frac{\partial v}{\partial \theta}+\frac{u}{r} \\
& \varepsilon_{\varphi}=\frac{1}{R+r \cos \theta} \frac{\partial w}{\partial \varphi}+\frac{\cos \theta}{R+r \cos \theta} u-\frac{\sin \theta}{R+r \cos \theta} v \\
& \gamma_{r \theta}=\frac{\partial v}{\partial r}-\frac{v}{r}+\frac{1}{r} \frac{\partial u}{\partial \theta}, \quad \gamma_{\theta \varphi}=\frac{1}{r} \frac{\partial w}{\partial \theta}+\frac{\sin \theta}{R+r \cos \theta} w+\frac{1}{R+r \cos \theta} \frac{\partial v}{\partial \varphi} \\
& \gamma_{\varphi r}=\frac{1}{R+r \cos \theta} \frac{\partial u}{\partial \varphi}+\frac{\partial w}{\partial r}-\frac{\cos \theta}{R+r \cos \theta} w
\end{aligned}
$$

Therefore, the strain energy $V$ and the kinetic energy $T$ of the shell panel undergoing free vibration are

$$
\begin{aligned}
& V=(1 / 2) \int_{0}^{\varphi_{0}} \int_{\theta_{0}}^{\theta_{0}+\theta_{1}} \int_{r_{0}}^{r_{1}}\left[(\lambda+2 G) \varepsilon_{r}^{2}+2 \lambda \varepsilon_{r} \varepsilon_{\theta}+2 \lambda \varepsilon_{r} \varepsilon_{\varphi}+(\lambda+2 G) \varepsilon_{\theta}^{2}+\right. \\
& \left.2 \lambda \varepsilon_{\theta} \varepsilon_{\varphi}+(\lambda+2 G) \varepsilon_{\varphi}^{2}+G\left(\gamma_{r \theta}^{2}+\gamma_{\theta \varphi}^{2}+\gamma_{\varphi r}^{2}\right)\right]|J| d r d \theta d \varphi, \\
& T=(\rho / 2) \int_{0}^{\varphi_{0}} \int_{\theta_{0}}^{\theta_{0}+\theta_{1}} \int_{r_{0}}^{r_{1}}\left(\dot{u}^{2}+\dot{v}^{2}+\dot{w}^{2}\right)|J| d r d \theta d \varphi
\end{aligned}
$$

where $\rho$ is the constant mass per unit volume; $\dot{u}, \dot{v}$ and $\dot{w}$ are the velocity components. The parameters $\lambda$ and $G$ are the Lamé constants for a homogeneous and isotropic material, which are expressed in terms of Young's modulus $E$ and Poisson's ratio $v$ by

$$
\lambda=v E /[(1+v)(1-2 v)] ; \quad G=E /[2(1+v)]
$$


In the free vibrations, the displacement components may be expressed as $u=U(\bar{r}, \bar{\theta}, \bar{\varphi}) e^{i \omega t}, \quad v=V(\bar{r}, \bar{\theta}, \bar{\varphi}) e^{i \omega t}, \quad w=W(\bar{r}, \bar{\theta}, \bar{\varphi}) e^{i \omega t}$

where $\omega$ is the circular eigenfrequency of the shell panel and $i=\sqrt{-1}$.

Defining the following dimensionless coordinates:

$$
\bar{R}=R / r_{1}, \quad \beta=r_{0} / r_{1}, \quad \bar{r}=\left(r-r_{0}\right) /\left(r_{1}-r_{0}\right), \quad \bar{\varphi}=\varphi / \varphi_{0}, \quad \nabla=\left(\theta-\theta_{0}\right) / \theta_{1}
$$

Substituting equations (6) and (7) into equation (4) gives the maximums of strain and kinetic energies:

$$
\begin{gathered}
V_{\max }=\frac{G}{2} r_{1} \theta_{1} \varphi_{0}(1-\beta) \int_{0}^{1} \int_{0}^{1} \int_{0}^{1}\left[(\bar{\lambda}+2) \bar{\varepsilon}_{r}^{2}+2 \bar{\lambda}_{\bar{\varepsilon}_{r}} \bar{\varepsilon}_{\theta}+2 \bar{\lambda}_{\bar{\varepsilon}_{r}} \bar{\varepsilon}_{\varphi}+(\bar{\lambda}+2) \bar{\varepsilon}_{\theta}^{2}+2 \bar{\lambda}_{\bar{\varepsilon}_{\theta}} \bar{\varepsilon}_{\varphi}+\right. \\
\left.(\bar{\lambda}+2) \bar{\varepsilon}_{\varphi}^{2}+\bar{\gamma}_{r \theta}^{2}+\bar{\gamma}_{\theta \varphi}^{2}+\bar{\gamma}_{\varphi r}^{2}\right]\left\{\bar{R}+[\beta+(1-\beta) \bar{r}] \cos \left(\theta_{0}+\theta_{1} \bar{\theta}\right)\right\}[\beta+(1-\beta) \bar{r}] d \bar{r} d \bar{\theta} d \bar{\varphi} \\
T_{\max }=\frac{\rho}{2} r_{1}^{3} \varphi_{0} \theta_{1}(1-\beta) \omega^{2} \int_{0}^{1} \int_{0}^{1} \int_{0}^{1}\left(U^{2}+V^{2}+W^{2}\right)\{\bar{R}+ \\
\left.[\beta+(1-\beta) \bar{r}] \cos \left(\theta_{0}+\theta_{1} \bar{\theta}\right)\right\}[\beta+(1-\beta) \bar{r}] d \bar{r} d \bar{\theta} d \bar{\varphi}
\end{gathered}
$$

in which,

$$
\begin{aligned}
& \bar{\lambda}=\frac{2 v}{1-2 v}, \quad \bar{\varepsilon}_{r}^{2}=\frac{1}{(1-\beta)^{2}}\left(\frac{\partial U}{\partial \bar{r}}\right)^{2} \\
& \bar{\varepsilon}_{\theta}^{2}=\frac{1}{[\beta+(1-\beta) \bar{r}]^{2}}\left[\left(\frac{\partial V}{\theta_{1} \partial \bar{\theta}}\right)^{2}+2 U \frac{\partial V}{\theta_{1} \partial \bar{\theta}}+U^{2}\right], \\
& \bar{\varepsilon}_{\varphi}^{2}=\frac{1}{\left\{\bar{R}+[\beta+(1-\beta) \bar{r}] \cos \left(\theta_{0}+\theta_{1} \bar{\theta}\right)\right\}^{2}}\left[\frac{1}{\varphi_{0}^{2}}\left(\frac{\partial W}{\partial \bar{\varphi}}\right)^{2}+\frac{2 \cos \left(\theta_{0}+\theta_{1} \bar{\theta}\right)}{\varphi_{0}} U \frac{\partial W}{\partial \bar{\varphi}}-\right. \\
& \left.\frac{2 \sin \left(\theta_{0}+\theta_{1} \bar{\theta}\right)}{\varphi_{0}} V \frac{\partial W}{\partial \bar{\varphi}}+\cos ^{2}\left(\theta_{0}+\theta_{1} \bar{\theta}\right) U^{2}-\sin \left(2 \theta_{0}+2 \theta_{1} \bar{\theta}\right) U V+\sin ^{2}\left(\theta_{0}+\theta_{1} \bar{\theta}\right) V^{2}\right], \\
& \bar{\varepsilon}_{r} \bar{\varepsilon}_{\theta}=\frac{1}{(1-\beta)[\beta+(1-\beta) \bar{r}]}\left(\frac{\partial U}{\partial \bar{r}} \frac{\partial V}{\theta_{1} \partial \bar{\theta}}+U \frac{\partial U}{\partial \bar{r}}\right), \\
& \bar{\varepsilon}_{\theta} \bar{\varepsilon}_{\varphi}=\frac{1}{[\beta+(1-\beta) \bar{r}]\left\{\bar{R}+[\beta+(1-\beta) \bar{r}] \cos \left(\theta_{0}+\theta_{1} \bar{\theta}\right)\right\}}\left[\frac{1}{\varphi_{0}}\left(\frac{\partial V}{\theta_{1} \partial \bar{\theta}} \frac{\partial W}{\partial \bar{\varphi}}+U \frac{\partial W}{\partial \bar{\varphi}}\right)+\right. \\
& \left.\cos \left(\theta_{0}+\theta_{1} \bar{\theta}\right)\left(U \frac{\partial V}{\theta_{1} \partial \bar{\theta}}+U^{2}\right)-\sin \left(\theta_{0}+\theta_{1} \bar{\theta}\right)\left(V \frac{\partial V}{\theta_{1} \partial \bar{\theta}}+U V\right)\right] \\
& \bar{\varepsilon}_{\varphi} \bar{\varepsilon}_{r}=\frac{1}{(1-\beta)\left\{\bar{R}+[\beta+(1-\beta) \bar{r}] \cos \left(\theta_{0}+\theta_{1} \bar{\theta}\right)\right\}}\left[\frac{1}{\varphi_{0}} \frac{\partial U}{\partial \bar{r}} \frac{\partial W}{\partial \bar{\varphi}}+\right. \\
& \left.\cos \left(\theta_{0}+\theta_{1} \bar{\theta}\right) U \frac{\partial U}{\partial \bar{r}}-\sin \left(\theta_{0}+\theta_{1} \bar{\theta}\right) \frac{\partial U}{\partial \bar{r}} V\right],
\end{aligned}
$$




$$
\begin{aligned}
& \bar{\gamma}_{r \theta}^{2}=\left(\frac{\partial V}{(1-\beta) \partial \bar{r}}\right)^{2}-\frac{2}{\beta+(1-\beta) \bar{r}} V \frac{\partial V}{(1-\beta) \partial \bar{r}}+\frac{2}{\beta+(1-\beta) \bar{r}} \frac{\partial U}{\theta_{1} \partial \bar{\theta}} \frac{\partial V}{(1-\beta) \partial \bar{r}}+ \\
& \frac{1}{[\beta+(1-\beta) \bar{r}]^{2}}\left[V^{2}-2 \frac{\partial U}{\theta_{1} \partial \bar{\theta}} V+\left(\frac{\partial U}{\theta_{1} \partial \bar{\theta}}\right)^{2}\right] \\
& \bar{\gamma}_{\theta \varphi}^{2}=\frac{1}{[\beta+(1-\beta) \bar{r}]^{2}}\left(\frac{\partial W}{\theta_{1} \partial \bar{\theta}}\right)^{2}+\frac{2}{[\beta+(1-\beta) \bar{r}]\left\{\bar{R}+[\beta+(1-\beta) \bar{r}] \cos \left(\theta_{0}+\theta_{1} \bar{\theta}\right\}\right.}( \\
& \left.\sin \left(\theta_{0}+\theta_{1} \bar{\theta}\right) W \frac{\partial W}{\theta_{1} \partial \bar{\theta}}+\frac{1}{\varphi_{0}} \frac{\partial V}{\partial \bar{\varphi}} \frac{\partial W}{\theta_{1} \partial \bar{\theta}}\right)+\frac{1}{\left\{\bar{R}+[\beta+(1-\beta) \bar{r}] \cos \left(\theta_{0}+\theta_{1} \bar{\theta}\right)\right\}^{2}}[ \\
& \left.\sin ^{2}\left(\theta_{0}+\theta_{1} \bar{\theta}\right) W^{2}+\frac{2 \sin \left(\theta_{0}+\theta_{1} \bar{\theta}\right)}{\varphi_{0}} \frac{\partial V}{\partial \bar{\varphi}} W+\frac{1}{\varphi_{0}^{2}}\left(\frac{\partial V}{\partial \bar{\varphi}}\right)^{2}\right], \\
& \bar{\gamma}_{\varphi r}^{2}=\left(\frac{\partial W}{(1-\beta) \partial \bar{r}}\right)^{2}+\frac{2}{\bar{R}+[\beta+(1-\beta) \bar{r}] \cos \left(\theta_{0}+\theta_{1} \bar{\theta}\right)}\left(\frac{1}{\varphi_{0}} \frac{\partial U}{\partial \bar{\varphi}} \frac{\partial W}{(1-\beta) \partial \bar{r}}-\right. \\
& \left.-\cos \left(\theta_{0}+\theta_{1} \bar{\theta}\right) W \frac{\partial W}{(1-\beta) \partial \bar{r}}\right)+\frac{1}{\left\{\bar{R}+[\beta+(1-\beta) \bar{r}] \cos \left(\theta_{0}+\theta_{1} \bar{\theta}\right)\right\}^{2}}\left[\frac{1}{\varphi_{0}^{2}}\left(\frac{\partial U}{\partial \bar{\varphi}}\right)^{2}-\right. \\
& \left.\frac{2 \cos \left(\theta_{0}+\theta_{1} \bar{\theta}\right)}{\varphi_{0}} \frac{\partial U}{\partial \bar{\varphi}} W+\cos ^{2}\left(\theta_{0}+\theta_{1} \bar{\theta}\right) W^{2}\right]
\end{aligned}
$$

The Lagrangian energy functional $\Pi$ of the shell panel is given by

$$
\Pi=T_{\max }-V_{\max }
$$

The displacement functions $U(\bar{r}, \theta, \bar{\varphi}), V(\bar{r}, \theta, \bar{\varphi})$ and $W(\bar{r}, \theta, \bar{\varphi})$ are expressed in terms of finite series as

$$
\begin{aligned}
& U(\bar{r}, \theta, \bar{\varphi})=C_{u}(\bar{\theta}) D_{u}(\bar{\varphi}) \sum_{i=1}^{I} \sum_{j=1}^{J} \sum_{k=1}^{K} A_{i j k} F_{i}(\bar{r}) H_{j}(\bar{\theta}) F_{k}(\bar{\varphi}), \\
& V(\bar{r}, \theta, \bar{\varphi})=C_{v}(\bar{\theta}) D_{v}(\bar{\varphi}) \sum_{l=1}^{L} \sum_{m=1}^{M} \sum_{n=1}^{N} B_{l m n} F_{l}(\bar{r}) H_{m}(\bar{\theta}) F_{n}(\bar{\varphi}) \\
& \bar{W}(\bar{r}, \theta, \bar{\varphi})=C_{w}(\bar{\theta}) D_{w}(\bar{\varphi}) \sum_{p=1}^{P} \sum_{q=1}^{Q} \sum_{s=1}^{S} C_{p q s} F_{p}(\bar{r}) H_{q}(\bar{\theta}) F_{s}(\bar{\varphi})
\end{aligned}
$$

where $C_{u}(\bar{\theta}), C_{v}(\bar{\theta})$ and $C_{w}(\bar{\theta})$ are the boundary functions in the $\theta$ direction, which describe the boundary conditions of the panel at edges $\theta=\theta_{0}$ and $\theta=\theta_{0}+\theta_{1}$. $D_{u}(\bar{\varphi}), D_{v}(\bar{\varphi})$ and $D_{w}(\bar{\varphi})$ are the boundary functions in the $\varphi$ direction, which describe the boundary conditions of the panel at edges $\varphi=0$ and $\varphi=\varphi_{0} \cdot A_{i j k}$, $B_{l m n}$ and $C_{p q s}$ are the undetermined coefficients and $I, J, K, L, M, N, P, \mathrm{Q}, S$ are the truncated orders of their corresponding series. $F_{i}(\bar{r}), F_{l}(\bar{r}), F_{p}(\bar{r}), H_{j}(\bar{\theta})$, 
$H_{m}(\bar{\theta}), H_{q}(\bar{\theta})$ and $F_{k}(\bar{\varphi}), F_{n}(\bar{\varphi}), F_{s}(\bar{\varphi})$ are the Chebyshev polynomials of first kind, which can be uniformly expressed as:

$$
F_{i}(\chi)=\cos [(i-1) \arccos (2 \chi-1)], \quad i=1,2,3, \ldots, \quad \chi=\bar{r}, \bar{\theta}, \bar{\varphi}
$$

It is noted that in using the Ritz method, the stress boundary conditions of the panels need not be satisfied in advance, but the geometric boundary conditions should be satisfied exactly. There is no displacement restraint on the curved surfaces of the panels at $r=r_{0}$ and $r=r_{1}$. Therefore, the boundary functions $C_{u}(\bar{\theta}), C_{v}(\bar{\theta}), C_{w}(\bar{\theta})$ and $D_{u}(\bar{\varphi}), D_{v}(\bar{\varphi}), D_{w}(\bar{\varphi})$ are sufficient to enable the displacement components $u$, $v$ and $w$ satisfying the geometric boundary conditions at boundaries $\theta=\theta_{0}$, $\theta=\theta_{0}+\theta_{1}$, and $\varphi=0, \varphi=\varphi_{0}$ respectively, which are listed in Table 1.

It should be mentioned that the Chebyshev polynomials has two distinct advantages. One is that $F_{i}(\chi)(i=1,2,3, \ldots)$ is a set of complete and orthogonal series in the interval $[-1,1]$, which is more stable in numerical computations than other admissible functions such as the simple algebraic polynomials [38,39]. The other advantage is that $F_{i}(\chi)(i=1,2,3, \ldots)$ can be expressed in a simple and unified form of cosine functions, which is easier for coding than the orthogonal recurrent polynomials constructed from the Schmidt process. It is obvious that the completeness and orthogonality of the admissible functions in $\theta$ and/or $\varphi$ directions have been destroyed by the boundary functions, except for the complete free panels. However, the boundary functions used here always take positive values in the panel domain. This means that the boundary functions are ineffective to the zero point distributions of the admissible functions within the panel domain, which are completely determined by the Chebyshev polynomials. Namely, the boundary functions can only adjust the amplitude of the Chebyshev polynomials in the panel domain. Therefore, the main properties of the Chebyshev polynomials are still reserved in the admissible functions. 
We can conclude that there is no frequency lost in the present analysis if enough terms of the admissible functions are used.

Minimizing functional (10) with respect to the coefficients of displacement functions, i.e.

$\frac{\partial \Pi}{\partial A_{i j k}}=0, \quad \frac{\partial \Pi}{\partial B_{l m n}}=0, \quad \frac{\partial \Pi}{\partial C_{p q s}}=0$

we have the following eigenfrequency equation:

$$
\left[\left(\begin{array}{ccc}
{\left[K_{u u}\right]} & {\left[K_{u v}\right]} & {\left[K_{u w}\right]} \\
& {\left[K_{v v}\right]} & {\left[K_{v w}\right]} \\
S y m & & {\left[K_{w w}\right]}
\end{array}\right)-\Omega^{2}\left(\begin{array}{ccc}
{\left[M_{u u}\right]} & {[0]} & {[0]} \\
& {\left[M_{v v}\right]} & {[0]} \\
S y m & & {\left[M_{w w}\right]}
\end{array}\right]\right)\left\{\begin{array}{l}
\{A\} \\
\{B\} \\
\{C\}
\end{array}\right\}=\left\{\begin{array}{l}
\{0\} \\
\{0\} \\
\{0\}
\end{array}\right\}
$$

where $\Omega=\omega a \sqrt{\rho / G}$, and

$$
\{A\}=\left\{\begin{array}{c}
A_{111} \\
A_{112} \\
\vdots \\
A_{11 K} \\
A_{121} \\
\vdots \\
A_{12 K} \\
\vdots \\
A_{1 J K} \\
\vdots \\
A_{I J K}
\end{array}\right\}, \quad\{B\}=\left\{\begin{array}{c}
B_{111} \\
B_{112} \\
\vdots \\
B_{11 N} \\
B_{121} \\
\vdots \\
B_{12 N} \\
\vdots \\
B_{1 M N} \\
\vdots \\
B_{L M N}
\end{array}\right\}, \quad\{C\}=\left\{\begin{array}{c}
C_{111} \\
C_{112} \\
\vdots \\
C_{11 S} \\
C_{121} \\
\vdots \\
C_{12 S} \\
\vdots \\
C_{1 Q S} \\
\vdots \\
C_{P Q S}
\end{array}\right\}
$$

Each elements in matrices $\left[K_{i j}\right]$ and $\left[M_{i j}\right](i, j=u, v, w)$ can be numerically evaluated by the Gaussian quadrature. Solving equation (14), total $I \times J \times K+L \times M \times N+P \times Q \times R$ eigenvalues and the corresponding modes can be obtained.

\section{Convergence and Comparison}

In order to validate the reliability of the proposed approach described above, it is necessary to conduct the convergence studies to determine the number of terms of 
Chebyshev polynomial series used in equation (25). The convergence study is based upon the fact that all the frequencies obtained by the Ritz method should converge to their exact values in an upper bound manner. It is obvious that improper or very slow convergence means that the displacement functions chosen are poor ones. Two typical shell panels with completely free boundaries are considered firstly. One is taken from the convex part of the hollow torus, which is a cap-shaped shell panel. The other is taken from the concave part of the hollow torus, which is a saddle-shaped shell panel. The radius ratio of these two shell panels is $R / r_{1}=1.2$, the thickness ratio is $r_{0} / r_{1}=0.8$, the toroidal angle of the shell panels is $\varphi_{0}=90^{\circ}$ and the subtended angle of the cross-section is $\theta_{1}=90^{\circ}$. For the cap-shaped shell panel, the initial angle of the cross-section is $\theta_{0}=-45^{\circ}$ and for the saddle-shaped shell panel, the initial angle of the cross-section is $\theta_{0}=135^{\circ}$. The Poisson' ratio is $v=0.3$. From these shells configurations, the vibration modes can be classified into the AA, AS, SA and SS ones where the capital letter "A" means antisymmetric while "S" means symmetric. The first capital letter is with respect to the $\varphi$ plane and the second is with respect to the $r-\theta$ plane. Table 2 and Table 3 give the first eight dimensionless frequencies of every mode classifications for these two shell panels where six zero frequencies for completely free shell panel are not included. To make the convergence study simplified, equal numbers of Chebyshev polynomial terms in every coordinates were taken for all the three displacement functions $U, V$ and $W$, although using unequal numbers of Chebyshev polynomial terms could provide the optimal computations. Five groups of different terms were checked. It is seen from Table 2 and Table 3 that with the increase of the number of terms, all of the frequencies monotonically decrease. Using $9 \times 9 \times 9$ terms of the Chebyshev polynomials give the same frequencies with five significant figures as those using $10 \times 10 \times 10$ terms of the Chebyshev polynomials. Even only using $5 \times 5 \times 5$ terms still guarantee a satisfied accuracy. 
A comparison study of the present 3-D Chebyshev-Ritz solutions with previously published 2-D and 3-D solutions is given in Table 4 for spherical shells panels with square planform from thin shells to thick shells. In order to be in keeping with the references, the dimensionless frequency $w a \sqrt{\Omega / E}$ is taken with a new set of size parameters: the mean radius $r_{m}$, the shell thickness $h$ and the side length of the square planform $a$. The Poisson's ratio is $v=0.3$. Two kinds of boundary conditions are considered: completely free $(F F F F)$ and fully clamped $(C C C C)$. The available results are from the first-order theory [26], the third-order theory [8], the higher-order theory [7] and the exact 3-D theory [35], respectively. It is observed from Table 4 that in general the present Chebyshev-Ritz solutions are in good agreement with those from different theories, however closer to the orthogonal polynomial-Ritz solutions which are also from the exact 3-D elasticity [35]. It is seen that with the increase of the shell thickness, the differences between the 3-D solutions and the 2-D solutions increase, especially for the fully clamped (CCCC) spherical shell panels.

It is well known that the finite element solutions can provide reliable results with large computational cost. The comparative study of the present solutions with those obtained by the finite element (FE) method is summarized in Tables 5-7 for three shell panels: two cap-shaped shell panels and a saddle-shaped shell panel. The shells are made of concrete with the elastic modulus $E=3.25 \times 10^{10} \mathrm{~Pa}$, per unit volume $\rho=2600$ $\mathrm{kg} / \mathrm{m}^{3}$ and the Poisson's ratio $v=0.2$. The tetrahedral solid elements with four nodes in software package ANSYS, 38424 elements with 212658 degree of freedom, were used for the numerical computations. In Table 5 and Table 7, the sizes of the shell panels are $R=80 \mathrm{~m}, r_{0}=40 \mathrm{~m}$ and $r_{1}=50 \mathrm{~m}$ while in Table 6 , the sizes of the shell panel are $R=18 \mathrm{~m}, r_{0}=40 \mathrm{~m}$ and $r_{1}=50 \mathrm{~m}$. These three shell panels have the different toroidal angles, subtended angles and initial angles. Three kinds of boundary conditions are considered: completely free $(F F F F)$, fully clamped $(C C C C)$ and clamped at two 
edges in $\varphi$ direction but free at two edges at $\theta$ direction $(C F C F)$. It is seen from Tables 5-7 that the present solutions are in good agreement with the finite element solutions. Looking through the data, one can find that the present results are always lower than the corresponding ones from finite element. This means that the present solutions have higher accuracy than the finite element solutions because both the methods provide the upper bound values of the exact solutions. Moreover, it is seen that for thick shell panels, the frequencies tend to huddle together. Therefore, in some cases a large number of vibration modes could be required when a thick shell is subjected to broadband excitations. For example, when the thick panel is subjected to a shock load, it is necessary to use a large number of vibration modes to make a realistic prediction of the dynamic response. The present method just satisfies such a requirement because the numerical stability can be guaranteed when a large number of Chebyshev polynomials are used in the computations.

\section{Numerical Results}

Having verified the convergence and accuracy of the present method, the effects of various size parameters such as the radius ratio $R / r_{1}$, thickness ratio $r_{0} / r_{1}$, toroidal angle $\varphi_{0}$ initial angle $\theta_{0}$ and subtended angle $\theta_{1}$ on frequencies were discussed. In the following study, the radius ratio $R / r_{1}=1.5$ and the Poisson' ratio $v=0.3$ are fixed. Tables 8-11 study the effect of thickness ratio $r_{0} / r_{1}$ on frequencies of shell panels with toroidal angle $\varphi_{0}=90^{\circ}$ and subtended angle $\theta_{1}=90^{\circ}$. Two kinds of shell panels are considered: a cap-shaped shell panel with the initial angle $\theta_{0}=-45^{\circ}$ and a saddle-shaped shell panel with the initial angle $\theta_{0}=135^{\circ}$. Two boundary conditions are checked: completely free $(F F F F)$ and clamped at $\varphi$ direction but free at $\theta$ direction $(C F C F)$. It is seen from Tables $8-11$ that in most cases, with the increase of the thickness ratio $r_{0} / r_{1}$ frequencies decrease. This means that the frequencies of thick 
shells are higher than those of thin shells. However, we can find exceptional cases for some very thick shell panels, e.g. the eighth AS mode for $r_{0} / r_{1}=0.6$ in Table 10 , the eighth AS and SS modes for $r_{0} / r_{1}=0.6,0.7$ and the eighth AA mode for $r_{0} / r_{1}=0.6$ in Table 11. Moreover, we can see that the effect of the shell thickness on frequencies of thin shell panels is higher than that on frequencies of thick shell panels.

Figures 4-16 study the effect of initial angle $\theta_{0}$ on firstsix non-zero frequencies of shell panels with different toroidal angle $\varphi_{0}$ and subtended angle $\theta_{1}$. The thickness ratio is fixed at $r_{0} / r_{1}=0.8$. Due to the varying initial angle no symmetry can be guaranteed in the $\theta$ direction, only the symmetry about $\varphi$ can be classified if the panels have symmetric boundary conditions in the toroidal direction. It is seen from Figures 4-7, 9-12 and 14-16 that as a whole, the frequencies increase with the increase of the initial angle $\theta_{0}$. However, for the FFFF panels with $\theta_{1}=180^{\circ}$ and $\varphi_{0}=90^{\circ}$ such a trend is not clear as shown in Figure 8. Especially, in Figure 13 we see the contrary trend for the panels with $\theta_{1}=360^{\circ}$ and $\varphi_{0}=180^{\circ}$. It should be noted that Figures $10-15$ correspond to the toroidal shells with a crack along the meridian while Figure 16 correspond to the complete toroidal shells with two cracks: one is along the meridian and the other cuts off the cross-section.

The first two or four mode shapes of various mode classifications for three typical doubly-curved shell panels with toroidal angle $\varphi_{0}=180$ and subtended angle $\theta_{1}=180^{\circ}$ are plotted in Figures 17-19. All the panels have the CFCF boundary conditions. Three different initial angles $\theta_{1}=-90^{\circ}, 90^{\circ}$ and $0^{\circ}$ are checked. Figure 17 is the mode shapes for a cap-shaped shell panel, Figure 18 is those for a saddle-shaped shell panel and Figure 19 is those for a sectorial-shaped shell panels. It is seen that each modes are generally a combination of flexural, extensional, shear and torsional deformations.

\section{Conclusion}


The Chebyshev-Ritz approach is developed for the three-dimensional vibration analysis of doubly-curved shell panels. The present shell panel model describes a lot of commonly used shell-structural components. The analysis is based on the small strain linear elasticity theory. Convergence and comparison studies verify the advantage of the present method in accuracy and computational cost. When a large number of frequencies need to be obtained the computational robustness can be guaranteed by using the Chebyshev polynomials as admissible functions due to the excellent properties of Chebyshev polynomials in numerical computations. The method is straightforward, but it is capable of determining a large number of frequencies with high accuracy as desired. Therefore the data presented in the analysis may be regarded as benchmark results against which 3-D results obtained by other methods, such as finite elements and finite differences, and 2-D shell theories may be compared to determine the accuracy of the latter. The effect of various size parameters, such as the radius ratio, thickness ratio, toroidal angle, subtended and initial angles on frequencies of shell panels are discussed in detail. Mode shapes show a combination of the flexural, extensional, shear and torsional deformations. 


\section{References}

[1] M. Melaragno, An Introduction to Shell Structures, Van Nostrand Reinhold, New York, 1991.

[2] A.E.H. Love, The Mathematical Theory of Elasticity, Cambridge University Press, London, 1934.

[3] E. Reissner, A new derivation of the equations of the deformation of elastic shells, American Journal of Mathematics 63 (1941) 177-184.

[4] L.H. Donnell, A new theory for the buckling of thin cylinders under axial compression and bending. Transactions of the ASME 56 (1934) 795-806.

[5] W. Flügge, Stresses in Shells. Springer-Verlag, Berlin, 1973.

[6] Reaz A. Chaudhuri, Humayun R.H. Kabir, Static and dynamic Fourier analysis of finite cross-ply doubly curved panels using classical shallow shell theories, Composite Structures 28 (1994) 73-91.

[7] J.N. Reddy, Exact solutions of moderately thick laminated shells, ASCE journal of Engineering Mechanics 110 (1983) 794-809.

[8] J.N. Reddy, C.F. Liu, A higher-order shear deformation theory for laminated elastic shells, International Journal of Engineering Science 23 (1985) 319-330.

[9] Hasan Biglari, Ali Asghar Jafari, Higher-order free vibrations of doubly-curved sandwich panels with flexible core based on refined three-layered theory, Composite Structures 92 (2010) 2685-2694.

[10] Sh. Hosseini-Hashemi, M. Fadaee, On the free vibration of moderately thick spherical shell panel-A new exact closed-form procedure. Journal of Sound and Vibration, 330, 4352-4367, 2011.

[11] S. Pradyumna, J.N. Bandyopadhyay, Free vibration analysis of functional graded curved panels using a higher-order finite element formulation, Journal of 
Sound and Vibration 318 (2008) 176-192.

[12] Francesco Tornabene, Erasmo Viola, Vibration analysis of spherical structural elements using the GDQ method, Computers and Mathematics with Applications 53 (2007) 1538-1560.

[13] X. Zhao, T.Y. Ng, K.M. Liew, Free vibration of two-side simply-supported laminated cylindrical panels via the mesh-free kp-Ritz method, International Journal of Mechanical Sciences 46 (2004) 123-142.

[14] K.M. Liew, C.W. Lim, S. Kitipornchai, Vibration of shallow shells: A review with bibliography, ASME Applied Mechanics Reviews 50 (1997) 431-443.

[15] C.W. Lim, S. Kitipornchai, K.M. Liew, Comparative accuracy of shallow and deep shell theories for vibration of cylindrical shells, Journal of Vibration and Control 3 (1997) 119-143.

[16] M.S. Quta, A.W. Leissa, Vibration of shallow shells with 2 adjacent edges clamped and the others free, Mechanics of Structures and Machines 21 (1993) 285-301.

[17] M.S. Quta, A.W. Leissa, Effects of edge constraints upon shallow shell frequencies, Thin-Walled Structures 14 (1992) 347-379.

[18] Y. Narita, A. Leissa, Vibrations of completely free shallow shells of curvilinear planform, ASME Journal of Applied Mechanics 53 (1986) 647-651.

[19] C.W. Lim, K.M. Liew, A pb-2 Ritz formulation for flexural vibration of shallow cylindrical shells of rectangular planform, Journal of Sound and Vibration 31 (1994) 1519-1536.

[20] K.M. Liew, C.W. Lim, Vibratory characteristics of cantilevered rectangular shallow shells of variable thickness, AIAA Journal 32 (1994) 387-396.

[21] K.M. Liew, C.W. Lim, Vibratory characteristics of doubly-curved shallow shells of curvilinear planform, ASCE Journal of Engineering Mechanics 121 (1995) 
203-213.

[22] K.M. Liew, C.W. Lim, Vibration of perforated doubly-curved shallow shells with rounded corners, International Journal of Solids and Structures, 31 (1994) $1519-1536$.

[23] K.M. Liew, C.W. Lim, Vibration of doubly-curved shallow shells, Acta Mechanica 114 (1996) 95-119.

[24] C.W. Lim, K.M. Liew, Vibration of moderately thick cylindrical shallow shells, Journal of the Acoustics Society of America, 100 (1996) 3665-3673.

[25] C.W. Lim, K.M. Liew, A higher order theory for vibration of shear deformable cylindrical shallow shells, International Journal of Mechanical Sciences 37 (1995) 277-295.

[26] K.M. Liew, C.W. Lim, A Ritz vibration analysis of doubly-curved rectangular shallow shells using a refined first-order theory, Computer Methods in Applied Mechanics and Engineering 127 (1995) 145-162.

[27] K.M. Liew, C.W. Lim, A Higher-order theory for vibration of doubly curved shallow shells, ASME Journal of Applied Mechanics, 63 (1996) 587-593.

[28] K.M. Liew, C.W. Lim, Vibration of thick doubly-curved stress free shallow shells of curvilinear planform, ASCE Journal of Engineering Mechanics 123 (1997) 413-421.

[29] A.W. Leissa, J.H. Kang, Three-dimensional vibration analysis of thick shells of revolution, ASCE Journal of Engineering Mechanics 125 (1999) 1365-1371.

[30] A.W. Leissa, J.H. Kang, Three-dimensional vibration analysis of paraboloidal shells, JSME International Journal Series C-Mechanical Systems Machine Elements and Manufacturing 45 (2002) 2-7.

[31] J.H. Kang, A.W. Leissa, Three-dimensional vibration analysis of thick hyperboloidal shells of revolution, Journal of Sound and Vibration 282 (2005) 
277-296.

[32] J.H. Kang, A.W. Leissa, Three-dimensional vibrations of thick spherical shell segments with variable thickness, International Journal of Solids and Structures 37 (2000) 4811-4823.

[33] P.G. Young, Application of a three-dimensional shell theory to the free vibration of shells arbitrarily deep in one direction. Journal of Sound and Vibration, 238, 257-269, 2000.

[34] O.G. McGee, S.C. Spry, A three-dimensional analysis of the spherical and toroidal elastic vibrations of thick-walled spherical bodies of revolution, International Journal for Numerical methods in Engineering 40 (1997) $1359-1382$.

[35] K.M. Liew, L. X. Peng, T.Y. Ng, Three-dimensional vibration analysis of spherical shell panels subjected to different boundary conditions. International Journal of Mechanical Sciences 44 (2002) 2103-2117.

[36] C.W. Lim, K.M. Liew, S. Kitipornchai, Vibration of open cylindrical shells: A three-dimensional elasticity approach, Journal of the Acoustics Society of America 104 (1998) 1436-1443.

[37] K.M. Liew, L.A. Bergman, T.Y. Ng, K.Y. Lam, Three-dimensional vibration of cylindrical shell panels-solution by continuum and discrete approaches, Computational Mechanics 26 (2000) 208-221.

[38] L. Fox, I.B. Parker, Chebyshev Polynomials in Numerical Analysis, Oxford University Press, London, 1968.

[39] D. Zhou, Three-dimensional Vibration Analysis of Structural Elements Using Chebyshev-Ritz Method, PhD thesis, University of Hong Kong, Hong Kong, 2003.

[40] D. Zhou, Y.K. Cheung, S.H. Lo \& F.T.K. Au, 3-D vibration analysis of thick, 
solid and hollow circular cylinders via Chebyshev-Ritz method, Computer Methods in Applied Mechanics and Engineering 192 (2003) 1575-1589.

[41] D. Zhou, S.H. Lo \& Y.K. Cheung, 3-D vibration analysis of annular sector plates using the Chebyshev-Ritz method, Journal of Sound and Vibration 320 (2009) 421-437.

[42] D. Zhou \& S.H. Lo, Three-dimensional vibrations of annular thick plates with linearly varying thickness, Archive of Applied Mechanics, 82 (2012) 111-135.

[43] D. Zhou, F.T.K. Au, S.H. Lo \& Y.K. Cheung, Three-dimensional vibration analysis of a torus with circular cross-section, Journal of the Acoustical Society of America 112 (2002) 2831-2840.

[44] D. Zhou, W. Liu, O.G. McGee III, On the three-dimensional vibrations of a hollow elastic torus of annular cross-section, Archive of Applied Mechanics 81 (2011) 473-487.

[45] D. Zhou, Y.K. Cheung, S.H. Lo, 3-D vibration analysis of circular rings with sectorial cross-sections. Journal of Sound and Vibration, 329 (2010) 1523-1535.

[46] D. Zhou, Y.K. Cheung, S.H. Lo, Three-dimensional vibration analysis of toroidal sectors with solid circular cross-section, ASME Journal of Applied Mechanics 77 (2010) 555-562. 
Table 1 The common boundary functions

\begin{tabular}{|c|c|c|c|c|c|c|}
\hline B. C. & $\mathrm{C}_{\mathrm{w}}(\theta)$ & $\mathcal{G}_{\mathrm{v}}(\theta)$ & $c_{w}(6)$ & $D_{w}(\phi)$ & $D_{v}(\phi)$ & $D_{w}(\bar{q})$ \\
\hline $\mathrm{C}-\mathrm{C}$ & $\theta(1-\theta)$ & $\theta(1-\theta)$ & $\theta(1-\theta)$ & $\varphi(1-\varphi)$ & $\phi(1-\phi)$ & $\varphi(1-\varphi)$ \\
\hline F-F & 1 & 1 & 1 & 1 & 1 & 1 \\
\hline $\mathrm{C}-\mathrm{F}$ & $\bar{\theta}$ & $\bar{\theta}$ & $\bar{\theta}$ & $\varphi$ & $\varphi$ & $\varphi$ \\
\hline F-C & $1-6$ & $1-8$ & $1-6$ & $1-\phi$ & $1-\varphi$ & $1-\phi$ \\
\hline
\end{tabular}

Note: B. C. means the boundary conditions in two opposite edges; C means the clamped edge; F means the free edge. The first capital letter is for the boundary condition at $\theta=\theta_{0}$ and for that at $\varphi=0$. The second capital letter is for the boundary condition at $\theta=\theta_{0}+\theta_{1}$ and for that at $\varphi=\varphi_{0}$. 
Table 2 The convergence study of the first eight non-zero dimensionless frequencies $\omega r_{1} \sqrt{\beta / G}$ of various mode classifications for a cap-shaped shell panel with the size parameters: $R / r_{1}=1.2, r_{0} / r_{1}=0.8, \varphi_{0}=90^{\circ}, \theta_{0}=-45^{\circ}, \theta_{1}=90^{\circ}$

\begin{tabular}{|c|c|c|c|c|c|c|c|c|}
\hline Terms & $\Omega_{1}$ & $\Omega_{2}$ & $\Omega_{3}$ & $\Omega_{4}$ & $\Omega_{5}$ & $\Omega_{6}$ & $\Omega_{7}$ & $\Omega_{8}$ \\
\hline \multicolumn{9}{|l|}{ AA mode } \\
\hline $5 \times 5 \times 5$ & 0.28105 & 1.0281 & 1.7731 & 2.1965 & 2.5767 & 2.6997 & 3.2336 & 3.8521 \\
\hline $6 \times 6 \times 6$ & 0.28100 & 1.0276 & 1.7731 & 2.1844 & 2.5735 & 2.6996 & 3.2298 & 3.8234 \\
\hline $7 \times 7 \times 7$ & 0.28098 & 1.0274 & 1.7731 & 2.1840 & 2.5733 & 2.6996 & 3.2291 & 3.7535 \\
\hline $8 \times 8 \times 8$ & 0.28098 & 1.0274 & 1.7731 & 2.1840 & 2.5732 & 2.6996 & 3.2290 & 3.7487 \\
\hline $9 \times 9 \times 9$ & 0.28098 & 1.0274 & 1.7731 & 2.1839 & 2.5732 & 2.6996 & 3.2289 & 3.7486 \\
\hline $10 \times 10 \times 10$ & 0.28098 & 1.0274 & 1.7731 & 2.1839 & 2.5732 & 2.6996 & 3.2289 & 3.7486 \\
\hline \multicolumn{9}{|l|}{ AS mode } \\
\hline $5 \times 5 \times 5$ & 0.68236 & 1.1582 & 1.9192 & 2.0108 & 3.0234 & 3.1494 & 3.6005 & 4.0807 \\
\hline $6 \times 6 \times 6$ & 0.68234 & 1.1579 & 1.9124 & 2.0054 & 3.0224 & 3.1354 & 3.4925 & 3.5993 \\
\hline $7 \times 7 \times 7$ & 0.68234 & 1.1578 & 1.9122 & 2.0051 & 3.0224 & 3.1350 & 3.4189 & 3.5991 \\
\hline $8 \times 8 \times 8$ & 0.68234 & 1.1578 & 1.9122 & 2.0050 & 3.0224 & 3.1348 & 3.4140 & 3.5990 \\
\hline $9 \times 9 \times 9$ & 0.68234 & 1.1578 & 1.9122 & 2.0050 & 3.0224 & 3.1348 & 3.4139 & 3.5990 \\
\hline $10 \times 10 \times 10$ & 0.68234 & 1.1578 & 1.9122 & 2.0050 & 3.0224 & 3.1348 & 3.4139 & 3.5990 \\
\hline \multicolumn{9}{|l|}{ SA mode } \\
\hline $5 \times 5 \times 5$ & 0.59626 & 1.1234 & 1.5622 & 2.4928 & 2.6529 & 2.8184 & 3.2289 & 3.5502 \\
\hline $6 \times 6 \times 6$ & 0.59579 & 1.1233 & 1.5592 & 2.4900 & 2.6527 & 2.8037 & 2.9974 & 3.5451 \\
\hline $7 \times 7 \times 7$ & 0.59568 & 1.1233 & 1.5588 & 2.4896 & 2.6526 & 2.8014 & 2.9797 & 3.5449 \\
\hline $8 \times 8 \times 8$ & 0.59566 & 1.1233 & 1.5587 & 2.4896 & 2.6526 & 2.8012 & 2.9791 & 3.5449 \\
\hline $9 \times 9 \times 9$ & 0.59565 & 1.1233 & 1.5587 & 2.4896 & 2.6526 & 2.8012 & 2.9791 & 3.5449 \\
\hline $10 \times 10 \times 10$ & 0.59565 & 1.1233 & 1.5587 & 2.4896 & 2.6526 & 2.8012 & 2.9791 & 3.5449 \\
\hline \multicolumn{9}{|l|}{ SS mode } \\
\hline $5 \times 5 \times 5$ & 0.26227 & 1.0273 & 1.3108 & 1.5264 & 1.7613 & 2.5286 & 2.8912 & 3.5355 \\
\hline $6 \times 6 \times 6$ & 0.26227 & 1.0271 & 1.3098 & 1.5251 & 1.7613 & 2.5221 & 2.6656 & 3.5314 \\
\hline $7 \times 7 \times 7$ & 0.26227 & 1.0270 & 1.3098 & 1.5249 & 1.7613 & 2.5212 & 2.6495 & 3.5310 \\
\hline $8 \times 8 \times 8$ & 0.26226 & 1.0270 & 1.3098 & 1.5248 & 1.7613 & 2.5210 & 2.6490 & 3.5310 \\
\hline $9 \times 9 \times 9$ & 0.26226 & 1.0270 & 1.3098 & 1.5248 & 1.7613 & 2.5210 & 2.6490 & 3.5310 \\
\hline $10 \times 10 \times 10$ & 0.26226 & 1.0270 & 1.3098 & 1.5248 & 1.7613 & 2.5210 & 2.6490 & 3.5310 \\
\hline
\end{tabular}


Table 3 The convergence study of the first eight non-zero dimensionless frequencies $\omega m_{1} \sqrt{\beta / Q}$ of various mode classifications for a saddle-shaped shell panel with the size parameters: $R / r_{1}=1.2, r_{0} / r_{1}=0.8, \varphi_{0}=90^{\circ}, \theta_{0}=135^{\circ}, \theta_{1}=90^{\circ}$

\begin{tabular}{|c|c|c|c|c|c|c|c|c|}
\hline Terms & $\Omega_{1}$ & $\Omega_{2}$ & $\Omega_{3}$ & $\Omega_{4}$ & $\Omega_{5}$ & $\Omega_{6}$ & $\Omega_{7}$ & $\Omega_{8}$ \\
\hline \multicolumn{9}{|l|}{ AA mode } \\
\hline $5 \times 5 \times 5$ & 0.94170 & 3.0243 & 4.2650 & 6.1973 & 6.5624 & 7.4153 & 8.9198 & 9.3255 \\
\hline $6 \times 6 \times 6$ & 0.94163 & 3.0239 & 4.2647 & 6.1930 & 6.5592 & 7.3810 & 8.8906 & 9.3237 \\
\hline $7 \times 7 \times 7$ & 0.94162 & 3.0239 & 4.2647 & 6.1928 & 6.5590 & 7.3796 & 8.8882 & 9.3235 \\
\hline $8 \times 8 \times 8$ & 0.94162 & 3.0238 & 4.2647 & 6.1928 & 6.5590 & 7.3794 & 8.8881 & 9.3235 \\
\hline $9 \times 9 \times 9$ & 0.94162 & 3.0238 & 4.2647 & 6.1928 & 6.5590 & 7.3794 & 8.8881 & 9.3235 \\
\hline $10 \times 10 \times 10$ & 0.94162 & 3.0238 & 4.2647 & 6.1928 & 6.5590 & 7.3794 & 8.8881 & 9.3235 \\
\hline \multicolumn{9}{|l|}{ AS mode } \\
\hline $5 \times 5 \times 5$ & 1.4435 & 3.0861 & 5.0080 & 5.8091 & 6.7904 & 7.7243 & 9.7483 & 10.086 \\
\hline $6 \times 6 \times 6$ & 1.4434 & 3.0859 & 5.0020 & 5.8074 & 6.7822 & 7.7087 & 9.2440 & 9.9925 \\
\hline $7 \times 7 \times 7$ & 1.4434 & 3.0858 & 5.0018 & 5.8073 & 6.7817 & 7.7077 & 9.2001 & 9.9842 \\
\hline $8 \times 8 \times 8$ & 1.4434 & 3.0858 & 5.0017 & 5.8073 & 6.7816 & 7.7077 & 9.1983 & 9.9837 \\
\hline $9 \times 9 \times 9$ & 1.4434 & 3.0858 & 5.0017 & 5.8073 & 6.7816 & 7.7077 & 9.1982 & 9.9837 \\
\hline $10 \times 10 \times 10$ & 1.4434 & 3.0858 & 5.0017 & 5.8073 & 6.7816 & 7.7077 & 9.1982 & 9.9837 \\
\hline \multicolumn{9}{|l|}{ SA mode } \\
\hline $5 \times 5 \times 5$ & 2.3202 & 3.4383 & 5.8373 & 6.4253 & 6.5956 & 7.7489 & 8.2973 & 9.3983 \\
\hline $6 \times 6 \times 6$ & 2.3199 & 3.4376 & 5.8353 & 6.3727 & 6.5941 & 7.7457 & 8.2897 & 9.3666 \\
\hline $7 \times 7 \times 7$ & 2.3199 & 3.4376 & 5.8352 & 6.3693 & 6.5940 & 7.7454 & 8.2888 & 9.3618 \\
\hline $8 \times 8 \times 8$ & 2.3199 & 3.4376 & 5.8352 & 6.3692 & 6.5940 & 7.7454 & 8.2888 & 9.3615 \\
\hline $9 \times 9 \times 9$ & 2.3199 & 3.4376 & 5.8352 & 6.3692 & 6.5940 & 7.7454 & 8.2888 & 9.3614 \\
\hline $10 \times 10 \times 10$ & 2.3199 & 3.4376 & 5.8352 & 6.3692 & 6.5940 & 7.7454 & 8.2888 & 9.3614 \\
\hline \multicolumn{9}{|l|}{ SS mode } \\
\hline $5 \times 5 \times 5$ & 0.84570 & 3.2513 & 3.5837 & 4.3456 & 5.7994 & 7.1231 & 7.4558 & 8.5158 \\
\hline $6 \times 6 \times 6$ & 0.84568 & 3.2508 & 3.5834 & 4.3371 & 5.7974 & 7.1191 & 7.4494 & 8.4167 \\
\hline $7 \times 7 \times 7$ & 0.84568 & 3.2507 & 3.5834 & 4.3368 & 5.7972 & 7.1188 & 7.4488 & 8.3899 \\
\hline $8 \times 8 \times 8$ & 0.84568 & 3.2507 & 3.5834 & 4.3368 & 5.7971 & 7.1187 & 7.4488 & 8.3882 \\
\hline $9 \times 9 \times 9$ & 0.84568 & 3.2507 & 3.5833 & 4.3368 & 5.7971 & 7.1187 & 7.4488 & 8.3881 \\
\hline $10 \times 10 \times 10$ & 0.84568 & 3.2507 & 3.5833 & 4.3368 & 5.7971 & 7.1187 & 7.4488 & 8.3881 \\
\hline
\end{tabular}


Table 4 The comparison study of the first three dimensionless frequencies $w a \sqrt{\Omega / E}$ of various mode classifications for spherical shell panels with square planform $\left(a / r_{m}=0.5, v=0.3\right)$

\begin{tabular}{|c|c|c|c|c|c|c|c|c|c|c|}
\hline$h / a$ & Ref. & SS-1 & $\mathrm{SS}-2$ & SS-3 & AS-1 & AS-2 & AS-3 & AA-1 & AA-2 & AA-3 \\
\hline \multicolumn{11}{|c|}{$F F F F$ spherical shell panels } \\
\hline \multirow[t]{2}{*}{0.01} & $\lceil 35\rceil$ & 0.060346 & 0.1503 & 0.4132 & 0.1126 & 0.2610 & 0.4695 & 0.041287 & 0.2136 & 0.3157 \\
\hline & Present & 0.060344 & 0.1502 & 0.4130 & 0.1125 & 0.2607 & 0.4690 & 0.041225 & 0.2134 & 0.3153 \\
\hline \multirow[t]{4}{*}{0.1} & $\lceil 26\rceil$ & 0.57042 & 0.7841 & 1.7244 & 0.9654 & 1.7084 & 2.6277 & 0.38434 & 1.8309 & 2.0684 \\
\hline & $\lceil 81$ & 0.56635 & 0.7799 & 1.7210 & 0.9621 & 1.7025 & 2.6283 & 0.38299 & 1.8272 & 2.0631 \\
\hline & $\lceil 35\rceil$ & 0.56477 & 0.7688 & 1.7153 & 0.9726 & 1.6719 & 2.6202 & 0.38566 & 1.8324 & 2.0605 \\
\hline & Present & 0.56477 & 0.7688 & 1.7152 & 0.9725 & 1.6719 & 2.6201 & 0.38565 & 1.8324 & 2.0604 \\
\hline \multirow[t]{2}{*}{0.2} & $\lceil 35\rceil$ & 1.0393 & 1.2984 & 2.7458 & 1.6751 & 2.6179 & 2.7158 & 0.70868 & 2.4336 & 2.9204 \\
\hline & Present & 1.0393 & 1.2984 & 2.7456 & 1.6751 & 2.6179 & 2.7155 & 0.70867 & 2.4336 & 2.9203 \\
\hline \multirow[t]{5}{*}{0.5} & $\lceil 26\rceil$ & 1.8691 & 2.2768 & 2.7555 & 2.5575 & 2.6627 & 3.4526 & 1.3089 & 2.4434 & 3.2452 \\
\hline & $\lceil 8\rceil$ & 1.8689 & 2.2706 & 2.7499 & 2.5500 & 2.7059 & 3.4492 & 1.3216 & 2.4367 & 3.2554 \\
\hline & $\lceil 7\rceil$ & 1.8759 & 2.2875 & 2.7524 & 2.5545 & 2.6794 & 3.4701 & 1.3142 & 2.4441 & 3.2577 \\
\hline & $\lceil 35\rceil$ & 1.8665 & 2.2390 & 2.7317 & 2.5254 & 2.6792 & 3.4627 & 1.3191 & 2.4199 & 3.2979 \\
\hline & Present & 1.8641 & 2.2347 & 2.7315 & 2.5231 & 2.6738 & 3.4529 & 1.3176 & 2.4198 & 3.2944 \\
\hline \multicolumn{11}{|c|}{$C C C C$ spherical shell panels } \\
\hline \multirow[t]{2}{*}{0.01} & $\lceil 35\rceil$ & 0.59165 & 0.6481 & 0.7754 & 0.5764 & 0.7268 & 0.8068 & 0.63061 & 0.8857 & 0.8996 \\
\hline & Present & 0.59125 & 0.6474 & 0.7748 & 0.5763 & 0.7258 & 0.8055 & 0.63032 & 0.8837 & 0.8981 \\
\hline \multirow[t]{4}{*}{0.1} & $\lceil 26\rceil$ & 1.2106 & 3.1471 & 3.1915 & 1.9447 & 3.7149 & 3.8243 & 2.6888 & 4.4380 & 5.1226 \\
\hline & $\lceil 8\rceil$ & 1.2005 & 3.1331 & 1.1782 & 1.9314 & 3.7025 & 3.8114 & 2.6749 & 4.4281 & 5.1086 \\
\hline & $\lceil 35\rceil$ & 1.1881 & 3.1075 & 3.1560 & 1.9150 & 3.6824 & 3.8029 & 2.6610 & 4.3726 & 5.1028 \\
\hline & Present & 1.1879 & 3.1067 & 3.1552 & 1.9146 & 3.6819 & 3.7900 & 2.6604 & 4.3726 & 5.1015 \\
\hline \multirow[t]{4}{*}{0.2} & $\lceil 26\rceil$ & 1.7638 & 4.3337 & 4.4078 & 2.8281 & 3.7653 & 5.1442 & 3.8062 & 4.4359 & 5.4412 \\
\hline & $\lceil 8\rceil$ & 1.7454 & 4.3091 & 4.3861 & 2.8046 & 3.7546 & 5.1212 & 3.7827 & 4.4243 & 5.4329 \\
\hline & $\lceil 35\rceil$ & 1.7358 & 4.3197 & 4.3994 & 2.8061 & 3.7392 & 5.1465 & 3.8044 & 4.3662 & 5.4149 \\
\hline & Present & 1.7353 & 4.3181 & 4.3977 & 2.8106 & 3.7387 & 5.1447 & 3.8030 & 4.3662 & 5.4141 \\
\hline \multirow[t]{5}{*}{0.5} & $\lceil 26\rceil$ & 2.3853 & 5.2157 & 5.2940 & 3.4958 & 3.7688 & 5.5703 & 4.3724 & 4.6591 & 5.3267 \\
\hline & $\lceil 8\rceil$ & 2.4717 & 5.6115 & 5.7153 & 3.6005 & 3.9270 & 5.5368 & 4.4137 & 4.9816 & 5.4175 \\
\hline & $\lceil 7\rceil$ & 2.4916 & 5.6523 & 5.7427 & 3.6173 & 3.9000 & 5.5959 & 4.3672 & 5.0286 & 5.3185 \\
\hline & $\lceil 35\rceil$ & 2.3880 & 5.2207 & 5.3021 & 3.4662 & 3.7772 & 5.5791 & 4.2762 & 4.6901 & 5.2486 \\
\hline & Present & 2.3855 & 5.2165 & 5.2971 & 3.4638 & 3.7750 & 5.5782 & 4.2761 & 4.6861 & 5.2460 \\
\hline
\end{tabular}


Table 5 The comparison study of the first forty frequencies $(\mathrm{Hz}) f_{i}(i=1,2, \ldots, 40)$ of the present 3-D solutions with the 3-D finite element solutions for a cap-shaped shell panel with the size parameters: $R / r_{1}=1.6, r_{0} / r_{1}=0.8, \theta_{0}=-45^{\circ}, \theta_{1}=90^{\circ}, \varphi_{0}=45^{\circ}$

\begin{tabular}{|c|c|c|c|c|c|c|}
\hline$i$ & $\mathrm{FE}$ & Present & FE & Present & $\mathrm{FE}$ & Present \\
\hline & \multicolumn{2}{|c|}{$F F F F$} & \multicolumn{2}{|c|}{$C C C C$} & \multicolumn{2}{|c|}{$C F C F$} \\
\hline 1 & 0 & 0 & 14.015 & $13.958^{\mathrm{SS}}$ & 6.2608 & $6.2293^{\mathrm{SS}}$ \\
\hline 2 & 0 & 0 & 16.581 & $16.418^{\mathrm{SA}}$ & 6.6338 & $6.5731^{\mathrm{SA}}$ \\
\hline 3 & 0 & 0 & 16.589 & $16.481^{\mathrm{AS}}$ & 10.270 & $10.232^{\mathrm{SA}}$ \\
\hline 4 & 0 & 0 & 21.638 & $21.429^{\mathrm{AA}}$ & 10.792 & $10.669^{\mathrm{SS}}$ \\
\hline 5 & 0 & 0 & 22.545 & $22.345^{\mathrm{SS}}$ & 10.905 & $10.805^{\mathrm{AS}}$ \\
\hline 6 & 0 & 0 & 23.793 & $23.778^{\mathrm{AS}}$ & 12.364 & $12.216^{\mathrm{AA}}$ \\
\hline 7 & 3.502 & $3.441^{\mathrm{AA}}$ & 28.143 & $27.882^{\mathrm{SA}}$ & 17.379 & $17.175^{\mathrm{AS}}$ \\
\hline 8 & 4.936 & $4.880^{\mathrm{SS}}$ & 28.962 & $28.894^{\mathrm{SA}}$ & 19.158 & $19.073^{\mathrm{SS}}$ \\
\hline 9 & 7.431 & $7.357^{\mathrm{SS}}$ & 29.469 & $29.153^{\mathrm{SS}}$ & 19.184 & $19.144^{\mathrm{AS}}$ \\
\hline 10 & 7.942 & $7.819^{\mathrm{SA}}$ & 30.365 & $30.063^{\mathrm{AS}}$ & 19.263 & $19.174^{\mathrm{AA}}$ \\
\hline 11 & 8.842 & $8.708^{\mathrm{AS}}$ & 31.767 & $31.746^{\mathrm{AA}}$ & 19.654 & $19.426^{\mathrm{SA}}$ \\
\hline 12 & 12.300 & $12.195^{\mathrm{AS}}$ & 32.853 & $32.488^{\mathrm{AS}}$ & 20.703 & $20.463^{\mathrm{SA}}$ \\
\hline 13 & 14.303 & $14.112^{\mathrm{AA}}$ & 36.087 & $35.712^{\mathrm{AA}}$ & 25.190 & $24.882^{\mathrm{AA}}$ \\
\hline 14 & 14.359 & $14.358^{\mathrm{SA}}$ & 37.550 & $37.226^{\mathrm{SS}}$ & 25.406 & $25.101^{\mathrm{SS}}$ \\
\hline 15 & 14.579 & $14.384^{\mathrm{SS}}$ & 38.379 & $38.356^{\mathrm{AA}}$ & 27.225 & $27.203^{\mathrm{AA}}$ \\
\hline 16 & 17.224 & $17.023^{\mathrm{SA}}$ & 38.645 & $38.348^{\mathrm{SS}}$ & 28.170 & $27.908^{\mathrm{AS}}$ \\
\hline 17 & 17.873 & $17.864^{\mathrm{AA}}$ & 39.734 & $39.715^{\mathrm{SA}}$ & 28.600 & $28.598^{\mathrm{SS}}$ \\
\hline 18 & 18.732 & $18.730^{\mathrm{SS}}$ & 40.318 & $40.104^{\mathrm{SS}}$ & 30.251 & $29.929^{\mathrm{AA}}$ \\
\hline 19 & 18.945 & $18.717^{\mathrm{AA}}$ & 43.124 & $42.672^{\mathrm{SA}}$ & 30.506 & $30.469^{\mathrm{AS}}$ \\
\hline 20 & 20.392 & $20.197^{\text {SS }}$ & 44.877 & $44.391^{\mathrm{AS}}$ & 31.351 & $31.328^{\mathrm{SA}}$ \\
\hline 21 & 21.354 & $21.088^{\mathrm{AS}}$ & 45.015 & $44.537^{\mathrm{SA}}$ & 32.191 & $31.850^{\mathrm{SS}}$ \\
\hline 22 & 22.524 & $22.250^{\mathrm{SA}}$ & 45.856 & $45.375^{\mathrm{AA}}$ & 32.669 & $32.272^{\mathrm{SA}}$ \\
\hline 23 & 23.827 & $23.530^{\mathrm{SA}}$ & 48.211 & $48.129^{\mathrm{AS}}$ & 34.388 & $33.980^{\mathrm{AS}}$ \\
\hline 24 & 24.935 & $24.920^{\mathrm{SS}}$ & 48.980 & $48.874^{\mathrm{AS}}$ & 36.247 & $36.035^{\mathrm{AS}}$ \\
\hline 25 & 25.035 & $25.020^{\mathrm{AS}}$ & 49.315 & $48.668^{\mathrm{SA}}$ & 36.451 & $36.227^{\mathrm{SS}}$ \\
\hline 26 & 27.909 & $27.900^{\mathrm{SA}}$ & 50.092 & $50.086^{\mathrm{AA}}$ & 37.520 & $37.206^{\mathrm{SS}}$ \\
\hline 27 & 28.459 & $28.442^{\mathrm{AS}}$ & 50.722 & $50.695^{\mathrm{SS}}$ & 39.237 & $39.234^{\mathrm{AS}}$ \\
\hline 28 & 28.865 & $28.837^{\mathrm{SS}}$ & 51.349 & $51.108^{\mathrm{SA}}$ & 39.964 & $39.787^{\mathrm{SA}}$ \\
\hline 29 & 29.063 & $28.857^{\mathrm{SS}}$ & 52.851 & $52.483^{\mathrm{SS}}$ & 40.181 & $39.868^{\mathrm{SS}}$ \\
\hline 30 & 29.206 & $28.968^{\mathrm{AA}}$ & 53.058 & $52.834^{\mathrm{AS}}$ & 41.214 & $40.708^{\mathrm{AA}}$ \\
\hline 31 & 29.792 & $29.496^{\mathrm{AS}}$ & 54.470 & $54.081^{\mathrm{AA}}$ & 41.358 & $41.307^{\mathrm{SA}}$ \\
\hline 32 & 30.277 & $29.973^{\mathrm{AA}}$ & 54.665 & $54.387^{\mathrm{SS}}$ & 42.753 & $42.245^{\mathrm{SS}}$ \\
\hline 33 & 30.667 & $30.361^{\mathrm{SS}}$ & 56.023 & $55.428^{\mathrm{AA}}$ & 43.514 & $43.485^{\mathrm{AA}}$ \\
\hline 34 & 31.717 & $31.368^{\mathrm{AS}}$ & 57.825 & $57.318^{\mathrm{SS}}$ & 44.208 & $43.693^{\mathrm{SS}}$ \\
\hline 35 & 32.509 & $32.159^{\mathrm{AA}}$ & 58.611 & $58.285^{\mathrm{AS}}$ & 46.435 & $45.980^{\mathrm{SA}}$ \\
\hline 36 & 35.492 & $35.075^{\mathrm{SS}}$ & 59.700 & $59.064^{\mathrm{SS}}$ & 46.919 & $46.846^{\mathrm{AS}}$ \\
\hline 37 & 36.931 & $36.762^{\mathrm{SA}}$ & 60.408 & $60.123^{\mathrm{AS}}$ & 47.648 & $47.648^{\mathrm{AA}}$ \\
\hline 38 & 37.083 & $36.912^{\mathrm{SS}}$ & 60.910 & $60.886^{\mathrm{SA}}$ & 48.479 & $47.994^{\mathrm{AS}}$ \\
\hline 39 & 37.656 & $37.390^{\mathrm{AS}}$ & 62.141 & $61.480^{\mathrm{AS}}$ & 49.848 & $49.332^{\mathrm{AA}}$ \\
\hline 40 & 38.273 & $38.076^{\mathrm{AS}}$ & 62.459 & $62.094^{\mathrm{SS}}$ & 50.474 & $49.858^{\mathrm{AS}}$ \\
\hline
\end{tabular}


Table 6 The comparison study of the first forty frequencies $(\mathrm{Hz}) f_{i}(i=1,2, \ldots, 40)$ of the present 3-D solutions with the 3-D finite element solutions for a cap-shaped shell panel with the size parameters: $R / r_{1}=0.36, r_{0} / r_{1}=0.8, \theta_{0}=-30^{\circ}, \theta_{1}=60^{\circ}, \varphi_{0}=60^{\circ}$

\begin{tabular}{|c|c|c|c|c|c|c|}
\hline$i$ & $\mathrm{FE}$ & Present & FE & Present & $\mathrm{FE}$ & Present \\
\hline & \multicolumn{2}{|c|}{$F F F F$} & \multicolumn{2}{|c|}{$C C C C$} & \multicolumn{2}{|c|}{$C F C F$} \\
\hline 1 & 0 & 0 & 21.197 & $21.082^{\mathrm{SS}}$ & 11.266 & $11.208^{\mathrm{SS}}$ \\
\hline 2 & 0 & 0 & 26.418 & $26.225^{\mathrm{AS}}$ & 12.971 & $12.872^{\mathrm{SA}}$ \\
\hline 3 & 0 & 0 & 31.425 & $31.188^{\mathrm{SA}}$ & 15.247 & $15.226^{\mathrm{SA}}$ \\
\hline 4 & 0 & 0 & 35.973 & $35.949^{\mathrm{AS}}$ & 18.431 & $18.267^{\mathrm{AS}}$ \\
\hline 5 & 0 & 0 & 38.009 & $37.687^{\mathrm{SS}}$ & 21.531 & $21.331^{\mathrm{SS}}$ \\
\hline 6 & 0 & 0 & 39.316 & $38.995^{\mathrm{AA}}$ & 22.372 & $22.152^{\mathrm{AA}}$ \\
\hline 7 & 7.234 & $7.1254^{\mathrm{AA}}$ & 42.694 & $42.636^{\mathrm{SA}}$ & 28.954 & $28.921^{\mathrm{AS}}$ \\
\hline 8 & 8.491 & $8.4005^{\mathrm{SS}}$ & 46.972 & $46.949^{\mathrm{AA}}$ & 29.179 & $29.131^{\mathrm{AA}}$ \\
\hline 9 & 15.406 & $15.262^{\mathrm{SA}}$ & 49.950 & $49.512^{\mathrm{SA}}$ & 32.427 & $32.091^{\mathrm{AS}}$ \\
\hline 10 & 15.461 & $15.274^{\mathrm{SS}}$ & 51.749 & $51.307^{\mathrm{AS}}$ & 33.333 & $33.033^{\mathrm{SS}}$ \\
\hline 11 & 18.360 & $18.139^{\mathrm{AS}}$ & 52.509 & $52.079^{\mathrm{SS}}$ & 36.879 & $36.509^{\mathrm{SA}}$ \\
\hline 12 & 21.542 & $21.362^{\mathrm{AS}}$ & 57.145 & $57.135^{\mathrm{AA}}$ & 38.094 & $37.783^{\mathrm{SA}}$ \\
\hline 13 & 22.064 & $22.049^{\mathrm{SA}}$ & 57.921 & $57.559^{\mathrm{AS}}$ & 40.257 & $40.236^{\mathrm{AA}}$ \\
\hline 14 & 26.365 & $26.268^{\mathrm{AA}}$ & 58.062 & $57.893^{\mathrm{SS}}$ & 40.569 & $40.553^{\mathrm{SS}}$ \\
\hline 15 & 27.379 & $27.164^{\mathrm{AA}}$ & 58.850 & $58.855^{\mathrm{SA}}$ & 43.662 & $43.626^{\mathrm{AS}}$ \\
\hline 16 & 27.943 & $27.880^{\mathrm{SS}}$ & 62.562 & $62.018^{\mathrm{AA}}$ & 45.534 & $45.065^{\mathrm{SS}}$ \\
\hline 17 & 28.850 & $28.582^{\mathrm{SS}}$ & 66.193 & $65.615^{\mathrm{SS}}$ & 46.616 & $46.225^{\mathrm{AA}}$ \\
\hline 18 & 34.146 & $33.879^{\mathrm{SA}}$ & 67.208 & $66.651^{\mathrm{sS}}$ & 46.701 & $46.565^{\mathrm{SA}}$ \\
\hline 19 & 35.224 & $35.222^{\mathrm{AS}}$ & 71.690 & $71.723^{\mathrm{AS}}$ & 48.905 & $48.495^{\mathrm{AS}}$ \\
\hline 20 & 35.432 & $35.409^{\mathrm{SS}}$ & 73.341 & $73.022^{\mathrm{SA}}$ & 52.507 & $52.008^{\mathrm{AA}}$ \\
\hline 21 & 36.275 & $35.991^{\mathrm{SS}}$ & 74.023 & $74.054^{\mathrm{AA}}$ & 53.233 & $53.178^{\mathrm{SS}}$ \\
\hline 22 & 36.847 & $36.521^{\mathrm{AA}}$ & 74.781 & $74.465^{\mathrm{SA}}$ & 57.444 & $57.302^{\mathrm{AS}}$ \\
\hline 23 & 40.236 & $39.778^{\mathrm{AS}}$ & 75.269 & $75.256^{\mathrm{SS}}$ & 57.578 & $57.535^{\mathrm{SS}}$ \\
\hline 24 & 40.938 & $40.931^{\mathrm{SS}}$ & 76.671 & $76.071^{\mathrm{SA}}$ & 58.289 & $57.700^{\mathrm{SA}}$ \\
\hline 25 & 41.241 & $40.961^{\mathrm{SA}}$ & 77.137 & $76.492^{\mathrm{AS}}$ & 59.230 & $58.838^{\mathrm{SS}}$ \\
\hline 26 & 41.361 & $41.341^{\mathrm{AS}}$ & 78.378 & $78.030^{\mathrm{AA}}$ & 60.322 & $59.834^{\mathrm{AS}}$ \\
\hline 27 & 41.374 & $41.230^{\mathrm{SA}}$ & 78.662 & $78.332^{\mathrm{AS}}$ & 60.711 & $60.676^{\mathrm{SA}}$ \\
\hline 28 & 43.348 & $43.328^{\mathrm{AA}}$ & 81.073 & $81.070^{\mathrm{SS}}$ & 64.114 & $64.002^{\mathrm{AA}}$ \\
\hline 29 & 44.999 & $44,548^{\mathrm{SA}}$ & 82.469 & $81.839^{\mathrm{AS}}$ & 64.952 & $64.433^{\mathrm{SS}}$ \\
\hline 30 & 52.346 & $51.976^{\mathrm{AS}}$ & 85.752 & $85.026^{\mathrm{SA}}$ & 65.509 & $64.990^{\mathrm{AS}}$ \\
\hline 31 & 52.586 & $52.017^{\mathrm{SS}}$ & 87.591 & $87.642^{\mathrm{AS}}$ & 68.689 & $68.069^{\mathrm{SA}}$ \\
\hline 32 & 54.201 & $54.147^{\mathrm{SS}}$ & 89.814 & $89.008^{\mathrm{SS}}$ & 69.213 & $69.091^{\mathrm{AS}}$ \\
\hline 33 & 54.780 & $54.243^{\mathrm{AA}}$ & 90.181 & $90.270^{\mathrm{SA}}$ & 70.282 & $70.254^{\mathrm{AA}}$ \\
\hline 34 & 56.063 & $56.043^{\mathrm{AS}}$ & 91.152 & $90.528^{\mathrm{AA}}$ & 71.905 & $71.220^{\mathrm{AA}}$ \\
\hline 35 & 56.450 & $56.398^{\mathrm{SS}}$ & 92.152 & $91.972^{\mathrm{AA}}$ & 73.696 & $73.334^{\mathrm{SS}}$ \\
\hline 36 & 56.763 & $56.391^{\mathrm{SA}}$ & 93.259 & $93.256^{\mathrm{AS}}$ & 74.732 & $74.032^{\mathrm{SS}}$ \\
\hline 37 & 57.114 & $57.097^{\mathrm{AS}}$ & 93.457 & $93.453^{\mathrm{SS}}$ & 74.884 & $74.915^{\mathrm{SA}}$ \\
\hline 38 & 57.565 & $57.129^{\mathrm{AA}}$ & 95.218 & $94.483^{\mathrm{AA}}$ & 76.353 & $75.955^{\mathrm{SS}}$ \\
\hline 39 & 58.088 & $57.894^{\mathrm{AS}}$ & 96.644 & $95.959^{\mathrm{SS}}$ & 77.546 & $77.567^{\mathrm{AA}}$ \\
\hline 40 & 58.297 & $57.955^{\mathrm{AA}}$ & 97.152 & $97.147^{\mathrm{AA}}$ & 79.022 & $79.004^{\mathrm{SA}}$ \\
\hline
\end{tabular}


Table 7 The comparison study of the first forty frequencies $(\mathrm{Hz}) f_{i}(i=1,2, \ldots, 40)$ of the present 3-D solutions with the 3-D finite element solutions for a saddle-shaped shell panel with the size parameters: $R / r_{1}=1.6, r_{0} / r_{1}=0.8, \theta_{0}=-135^{\circ}, \theta_{1}=90^{\circ}, \varphi_{0}=90^{\circ}$

\begin{tabular}{|c|c|c|c|c|c|c|}
\hline$i$ & FE & Present & FE & Present & FE & Present \\
\hline & \multicolumn{2}{|c|}{$F F F F$} & \multicolumn{2}{|c|}{ СССС } & \multicolumn{2}{|c|}{$C F C F$} \\
\hline 1 & 0.000 & 0.000 & 19.992 & $19.922^{\mathrm{SS}}$ & 11.505 & $11.458^{\mathrm{SA}}$ \\
\hline 2 & 0.000 & 0.000 & 22.041 & $21.913^{\mathrm{SA}}$ & 13.026 & $12.966^{\mathrm{SS}}$ \\
\hline 3 & 0.000 & 0.000 & 25.653 & $25.495^{\mathrm{AS}}$ & 16.700 & $16.634^{\mathrm{SS}}$ \\
\hline 4 & 0.000 & 0.000 & 29.673 & $29.468^{\mathrm{AA}}$ & 17.333 & $17.186^{\mathrm{AA}}$ \\
\hline 5 & 0.000 & 0.000 & 32.972 & $32.744^{\mathrm{SS}}$ & 17.660 & $17.518^{\mathrm{AS}}$ \\
\hline 6 & 0.000 & 0.000 & 34.895 & $34.868^{\mathrm{SA}}$ & 19.043 & $19.027^{\mathrm{SA}}$ \\
\hline 7 & 4.572 & $4.508^{\mathrm{AA}}$ & 37.165 & $37.045^{\mathrm{AS}}$ & 23.118 & $22.967^{\mathrm{AS}}$ \\
\hline 8 & 7.116 & $7.059^{\mathrm{SS}}$ & 39.623 & $39.426^{\mathrm{AS}}$ & 23.409 & $23.259^{\mathrm{SA}}$ \\
\hline 9 & 9.266 & $9.165^{\mathrm{AS}}$ & 41.509 & $41.198^{\mathrm{SS}}$ & 28.723 & $28.593^{\mathrm{AA}}$ \\
\hline 10 & 10.121 & $10.009^{\mathrm{SA}}$ & 43.113 & $42.790^{\mathrm{SA}}$ & 30.280 & $30.260^{\mathrm{SS}}$ \\
\hline 11 & 10.215 & $10.150^{\mathrm{SS}}$ & 44.857 & $44.818^{\mathrm{AA}}$ & 30.401 & $30.234^{\mathrm{AS}}$ \\
\hline 12 & 16.532 & $16.375^{\mathrm{SS}}$ & 46.001 & $45.685^{\mathrm{SA}}$ & 30.653 & $30.380^{\mathrm{SA}}$ \\
\hline 13 & 17.877 & $17.745^{\mathrm{SA}}$ & 47.908 & $47.798^{\mathrm{AA}}$ & 30.950 & $30.801^{\mathrm{SS}}$ \\
\hline 14 & 18.577 & $18.427^{\mathrm{AA}}$ & 49.782 & $49.432^{\mathrm{SS}}$ & 31.942 & $31.855^{\mathrm{AA}}$ \\
\hline 15 & 20.321 & $20.162^{\mathrm{AS}}$ & 52.109 & $51.841^{\mathrm{AA}}$ & 35.007 & $34.782^{\mathrm{SS}}$ \\
\hline 16 & 20.878 & $20.752^{\mathrm{AA}}$ & 53.773 & $53.716^{\mathrm{SS}}$ & 36.126 & $36.040^{\mathrm{AA}}$ \\
\hline 17 & 21.544 & $21.410^{\mathrm{AA}}$ & 56.231 & $56.148^{\mathrm{AS}}$ & 38.804 & $38.736^{\mathrm{AS}}$ \\
\hline 18 & 21.866 & $21.805^{\mathrm{AS}}$ & 57.761 & $57.372^{\mathrm{AS}}$ & 40.352 & $40.080^{\mathrm{SS}}$ \\
\hline 19 & 24.547 & $24.392^{\mathrm{SA}}$ & 58.331 & $57.890^{\mathrm{AA}}$ & 41.233 & $40.979^{\mathrm{AS}}$ \\
\hline 20 & 25.201 & $25.194^{\mathrm{SS}}$ & 59.338 & $58.941^{\mathrm{SA}}$ & 42.649 & $42.383^{\mathrm{SA}}$ \\
\hline 21 & 27.861 & $27.776^{\mathrm{SA}}$ & 60.089 & $59.689^{\mathrm{SS}}$ & 44.219 & $43.841^{\mathrm{AS}}$ \\
\hline 22 & 29.889 & $29.816^{\mathrm{AS}}$ & 62.242 & $61.995^{\mathrm{AS}}$ & 44.369 & $43.976^{\mathrm{AA}}$ \\
\hline 23 & 30.032 & $29.758^{\mathrm{SS}}$ & 63.647 & $63.622^{\mathrm{SA}}$ & 46.729 & $46.635^{\mathrm{AS}}$ \\
\hline 24 & 31.500 & $31.368^{\mathrm{SS}}$ & 63.676 & $63.664^{\mathrm{SS}}$ & 48.542 & $48.254^{\mathrm{SA}}$ \\
\hline 25 & 32.007 & $31.963^{\mathrm{SS}}$ & 64.714 & $64.256^{\mathrm{AS}}$ & 50.682 & $50.496^{\mathrm{SS}}$ \\
\hline 26 & 32.563 & $32.279^{\mathrm{AS}}$ & 67.098 & $66.854^{\mathrm{AS}}$ & 51.060 & $50.944^{\mathrm{SA}}$ \\
\hline 27 & 33.814 & $33.522^{\mathrm{SA}}$ & 67.206 & $67.146^{\mathrm{SA}}$ & 51.721 & $51.687^{\mathrm{SA}}$ \\
\hline 28 & 34.061 & $33.773^{\mathrm{SS}}$ & 69.937 & $69.815^{\mathrm{AA}}$ & 52.786 & $52.625^{\mathrm{AA}}$ \\
\hline 29 & 34.084 & $33.907^{\mathrm{AA}}$ & 70.816 & $70.739^{\mathrm{SS}}$ & 53.277 & $53.110^{\mathrm{SS}}$ \\
\hline 30 & 35.515 & $35.454^{\mathrm{AS}}$ & 71.843 & $71.350^{\mathrm{SS}}$ & 54.455 & $54.242^{\mathrm{SS}}$ \\
\hline 31 & 35.740 & $35.645^{\mathrm{SA}}$ & 72.577 & $72.201^{\mathrm{AA}}$ & 55.432 & $55.361^{\mathrm{SA}}$ \\
\hline 32 & 38.619 & $38.290^{\mathrm{SS}}$ & 73.798 & $73.460^{\mathrm{SS}}$ & 55.644 & $55.367^{\mathrm{AA}}$ \\
\hline 33 & 41.567 & $41.353^{\mathrm{AA}}$ & 73.974 & $73.264^{\mathrm{SA}}$ & 57.261 & $56.907^{\mathrm{AS}}$ \\
\hline 34 & 43.528 & $43.386^{\mathrm{AS}}$ & 74.369 & $73.863^{\mathrm{SA}}$ & 57.361 & $57.136^{\mathrm{AA}}$ \\
\hline 35 & 44.669 & $44.486^{\mathrm{SA}}$ & 77.702 & $77.692^{\mathrm{SA}}$ & 58.822 & $58.436^{\mathrm{SA}}$ \\
\hline 36 & 45.860 & $45.621^{\mathrm{sS}}$ & 77.938 & $77.449^{\mathrm{AA}}$ & 58.893 & $58.342^{\mathrm{SS}}$ \\
\hline 37 & 45.973 & $45.745^{\mathrm{SA}}$ & 79.946 & $79.636^{\mathrm{SS}}$ & 63.224 & $62.858^{\mathrm{SS}}$ \\
\hline 38 & 46.161 & $45.778^{\mathrm{AA}}$ & 81.703 & $81.244^{\mathrm{AS}}$ & 63.756 & $63.537^{\mathrm{SA}}$ \\
\hline 39 & 47.732 & $47.332^{\mathrm{AS}}$ & 81.933 & $81.832^{\mathrm{AA}}$ & 63.988 & $63.612^{\mathrm{AA}}$ \\
\hline 40 & 47.919 & $47.761^{\mathrm{SS}}$ & 83.260 & $82.917^{\mathrm{SS}}$ & 64.365 & $64.085^{\mathrm{AS}}$ \\
\hline
\end{tabular}


Table 8 The first eight non-zero dimensionless frequencies $\omega r_{1} \sqrt{R / \sqrt{g}}$ of various mode classifications for a $F F F F$ cap-shaped shell panel with the size parameters: $R / r_{1}=1.5, \varphi_{0}=90^{\circ}, \theta_{0}=-45^{\circ}, \theta_{1}=90^{\circ}$

\begin{tabular}{|c|c|c|c|c|c|c|c|c|}
\hline$r_{0} / r_{1}$ & $\Omega_{1}$ & $\Omega_{2}$ & $\Omega_{3}$ & $\Omega_{4}$ & $\Omega_{5}$ & $\Omega_{6}$ & $\Omega_{7}$ & $\Omega_{8}$ \\
\hline \multicolumn{9}{|c|}{ AA mode } \\
\hline 0.6 & 0.48588 & 1.4747 & 1.6943 & 2.7146 & 2.9461 & 3.4903 & 4.2472 & 4.3688 \\
\hline 0.7 & 0.36612 & 1.2344 & 1.5622 & 2.4311 & 2.6168 & 3.4191 & 3.5424 & 3.9634 \\
\hline 0.8 & 0.24172 & 0.86187 & 1.5239 & 1.7897 & 2.5072 & 2.5393 & 2.9941 & 3.1080 \\
\hline 0.9 & 0.11870 & 0.45899 & 1.0138 & 1.3434 & 1.4979 & 1.6991 & 1.8233 & 2.3006 \\
\hline 0.95 & 0.059081 & 0.25652 & 0.57800 & 0.74004 & 1.0177 & 1.0963 & 1.3897 & 1.4868 \\
\hline 0.99 & 0.011868 & 0.064738 & 0.13659 & 0.19300 & 0.31773 & 0.43933 & 0.51000 & 0.67273 \\
\hline \multicolumn{9}{|c|}{ AS mode } \\
\hline 0.6 & 0.84297 & 2.1241 & 2.3930 & 2.8242 & 3.0227 & 3.8102 & 4.1743 & 4.3730 \\
\hline 0.7 & 0.69580 & 1.6191 & 1.9961 & 2.4248 & 2.7926 & 3.5141 & 3.5877 & 3.7850 \\
\hline 0.8 & 0.54286 & 1.0980 & 1.5564 & 1.7761 & 2.6982 & 2.7489 & 2.7731 & 3.5541 \\
\hline 0.9 & 0.34283 & 0.60031 & 1.0369 & 1.1111 & 1.7005 & 1.7766 & 2.3541 & 2.4485 \\
\hline 0.95 & 0.18831 & 0.33106 & 0.64144 & 0.87642 & 1.0807 & 1.2926 & 1.3433 & 1.5000 \\
\hline 0.99 & 0.042373 & 0.077760 & 0.17497 & 0.27061 & 0.33930 & 0.48517 & 0.63785 & 0.68334 \\
\hline \multicolumn{9}{|c|}{ SA mode } \\
\hline 0.6 & 0.81916 & 1.1237 & 2.2008 & 2.4130 & 3.3915 & 3.8796 & 4.3685 & 4.6259 \\
\hline 0.7 & 0.71773 & 0.98240 & 1.7985 & 2.3430 & 3.1385 & 3.2852 & 3.5254 & 3.7949 \\
\hline 0.8 & 0.50631 & 0.93448 & 1.2960 & 2.3015 & 2.3482 & 2.5121 & 2.7495 & 3.1965 \\
\hline 0.9 & 0.26044 & 0.71082 & 0.91861 & 1.2851 & 1.4085 & 1.5147 & 1.9813 & 2.2642 \\
\hline 0.95 & 0.13736 & 0.40973 & 0.68567 & 0.85760 & 0.89171 & 0.92654 & 1.1938 & 1.3491 \\
\hline 0.99 & 0.031294 & 0.10569 & 0.15692 & 0.25072 & 0.38651 & 0.46814 & 0.58433 & 0.60815 \\
\hline \multicolumn{9}{|c|}{ SS mode } \\
\hline 0.6 & 0.32076 & 1.5185 & 1.5902 & 2.1053 & 2.5264 & 3.3710 & 3.5305 & 3.7604 \\
\hline 0.7 & 0.26174 & 1.2603 & 1.5491 & 1.5968 & 1.9743 & 2.7331 & 2.9665 & 3.6446 \\
\hline 0.8 & 0.20490 & 0.93470 & 1.1230 & 1.3940 & 1.5416 & 2.1244 & 2.2313 & 3.2594 \\
\hline 0.9 & 0.13377 & 0.50702 & 0.75093 & 0.85369 & 1.3720 & 1.4187 & 1.5118 & 2.0184 \\
\hline 0.95 & 0.076864 & 0.26390 & 0.46043 & 0.67606 & 0.85085 & 1.1227 & 1.2617 & 1.3619 \\
\hline 0.99 & 0.018060 & 0.056751 & 0.12087 & 0.23054 & 0.29639 & 0.40685 & 0.56727 & 0.60446 \\
\hline
\end{tabular}


Table 9 The first eight dimensionless frequencies $w r_{1} \sqrt{\Omega / G}$ of various mode classifications for a $C F C F$ cap-shaped shell panel with the size parameters: $R / r_{1}=1.5$, $\varphi_{0}=90^{\circ}, \theta_{0}=-45^{\circ}, \theta_{1}=90^{\circ}$

\begin{tabular}{|c|c|c|c|c|c|c|c|c|}
\hline$r_{0} / r_{1}$ & $\Omega_{1}$ & $\Omega_{2}$ & $\Omega_{3}$ & $\Omega_{4}$ & $\Omega_{5}$ & $\Omega_{6}$ & $\Omega_{7}$ & $\Omega_{8}$ \\
\hline \multicolumn{9}{|c|}{ AA mode } \\
\hline 0.6 & 1.1542 & 1.4084 & 2.5536 & 2.9159 & 3.1823 & 4.1795 & 4.6812 & 4.7041 \\
\hline 0.7 & 1.0100 & 1.3025 & 2.1908 & 2.8032 & 3.0902 & 3.6597 & 3.8593 & 4.5945 \\
\hline 0.8 & 0.77496 & 1.2572 & 1.6580 & 2.7107 & 2.8165 & 2.8832 & 3.0209 & 3.5931 \\
\hline 0.9 & 0.52185 & 0.97960 & 1.2369 & 1.5761 & 1.7411 & 2.1089 & 2.6259 & 2.7049 \\
\hline 0.95 & 0.39225 & 0.63568 & 0.97065 & 1.0408 & 1.2283 & 1.2998 & 1.5958 & 1.7191 \\
\hline 0.99 & 0.15394 & 0.28354 & 0.41874 & 0.52548 & 0.65699 & 0.68464 & 0.75394 & 0.83842 \\
\hline \multicolumn{9}{|c|}{ AS mode } \\
\hline 0.6 & 0.7426 & 1.5460 & 2.2051 & 2.6398 & 3.7709 & 3.7955 & 3.9251 & 4.4984 \\
\hline 0.7 & 0.62578 & 1.4860 & 1.9287 & 2.0965 & 3.1975 & 3.3231 & 3.7890 & 4.3637 \\
\hline 0.8 & 0.49923 & 1.3425 & 1.5263 & 1.6453 & 2.4463 & 2.6745 & 3.5885 & 3.6628 \\
\hline 0.9 & 0.35898 & 0.93177 & 0.98581 & 1.4510 & 1.5965 & 1.7681 & 2.2422 & 2.6108 \\
\hline 0.95 & 0.26248 & 0.59561 & 0.77688 & 1.0375 & 1.2175 & 1.4418 & 1.4779 & 1.6107 \\
\hline 0.99 & 0.11470 & 0.24041 & 0.36266 & 0.49415 & 0.65623 & 0.66105 & 0.72632 & 0.81774 \\
\hline \multicolumn{9}{|c|}{ SA mode } \\
\hline 0.6 & 0.60360 & 0.85013 & 1.8426 & 2.1570 & 3.3401 & 3.7777 & 4.1475 & 4.4569 \\
\hline 0.7 & 0.59876 & 0.75722 & 1.5650 & 2.0773 & 2.8990 & 3.6239 & 3.6751 & 4.0450 \\
\hline 0.8 & 0.58312 & 0.66618 & 1.1761 & 2.0384 & 2.2398 & 2.5947 & 3.1581 & 3.5421 \\
\hline 0.9 & 0.48227 & 0.60267 & 0.78297 & 1.3407 & 1.4219 & 1.8114 & 2.0088 & 2.1960 \\
\hline 0.95 & 0.32289 & 0.58483 & 0.63760 & 0.85511 & 0.87456 & 1.11184 & 1.3113 & 1.5005 \\
\hline 0.99 & 0.12041 & 0.24099 & 0.38062 & 0.53994 & 0.56162 & 0.61436 & 0.66952 & 0.72508 \\
\hline \multicolumn{9}{|c|}{ SS mode } \\
\hline 0.6 & 0.66648 & 1.4655 & 2.2701 & 2.8111 & 3.0357 & 3.1741 & 4.2738 & 4.4281 \\
\hline 0.7 & 0.62627 & 1.2578 & 1.7375 & 2.5331 & 2.6050 & 2.8814 & 3.8546 & 4.0903 \\
\hline 0.8 & 0.58225 & 1.0194 & 1.2254 & 1.9439 & 2.0426 & 2.7887 & 2.9995 & 3.3337 \\
\hline 0.9 & 0.49563 & 0.76718 & 0.78704 & 1.2583 & 1.3413 & 1.8884 & 2,1433 & 2.4927 \\
\hline 0.95 & 0.35682 & 0.61849 & 0.65596 & 0.84246 & 1.0306 & 1.3178 & 1.3643 & 1.4472 \\
\hline 0.99 & 0.15005 & 0.26700 & 0.39427 & 0.54250 & 0.57705 & 0.65252 & 0.65871 & 0.74141 \\
\hline
\end{tabular}


Table 10 The first eight dimensionless frequencies $\Delta r_{1} \sqrt{\rho / \sqrt{G}}$ of various mode classifications for a FFFF saddle-shaped shell panel with the size parameters: $R / r_{1}=1.5, \varphi_{0}=90^{\circ}, \theta_{0}=135^{\circ}, \theta_{1}=90^{\circ}$

\begin{tabular}{|c|c|c|c|c|c|c|c|c|}
\hline$r_{0} / r_{1}$ & $\Omega_{1}$ & $\Omega_{2}$ & $\Omega_{3}$ & $\Omega_{4}$ & $\Omega_{5}$ & $\Omega_{6}$ & $\Omega_{7}$ & $\Omega_{8}$ \\
\hline \multicolumn{9}{|c|}{ AA mode } \\
\hline 0.6 & 1.2877 & 3.0622 & 4.1476 & 4.8669 & 5.6752 & 6.9662 & 7.1526 & 7.6842 \\
\hline 0.7 & 0.99611 & 3.0454 & 3.6811 & 4.2736 & 5.4905 & 6.7931 & 7.4639 & 7.6048 \\
\hline 0.8 & 0.68612 & 2.6981 & 3.1511 & 3.5384 & 5.1786 & 6.1769 & 6.6304 & 7.3458 \\
\hline 0.9 & 0.35463 & 1.5674 & 2.2909 & 3.1058 & 4.0005 & 4.1310 & 5.2863 & 5.7693 \\
\hline 0.95 & 0.18061 & 0.88207 & 1.3969 & 2.3479 & 2.4336 & 3.1209 & 3.5126 & 3.8059 \\
\hline 0.99 & 0.036559 & 0.22856 & 0.42907 & 0.80368 & 0.97050 & 1.07372 & 1.1990 & 1.3255 \\
\hline \multicolumn{9}{|c|}{ AS mode } \\
\hline 0.6 & 2.4286 & 3.5524 & 4.3761 & 5.0720 & 6.1122 & 6.7177 & 6.9329 & 7.7360 \\
\hline 0.7 & 1.9011 & 3.3070 & 4.0958 & 4.9821 & 5.8285 & 6.0219 & 6.8410 & 7.9567 \\
\hline 0.8 & 1.3492 & 2.9868 & 3.4899 & 4.6408 & 4.8793 & 5.3541 & 6.5644 & 7.5486 \\
\hline 0.9 & 0.73106 & 2.2551 & 2.6886 & 2.7869 & 3.7436 & 4.8879 & 5.0551 & 5.5713 \\
\hline 0.95 & 0.39251 & 1.3883 & 1.5574 & 2.3526 & 2.6389 & 3.0248 & 3.2611 & 3.5671 \\
\hline 0.99 & 0.092176 & 0.34862 & 0.61857 & 0.87822 & 0.98878 & 1.1049 & 1.4298 & 1.4876 \\
\hline \multicolumn{9}{|c|}{ SA mode } \\
\hline 0.6 & 2.4240 & 3.8423 & 4.2395 & 4.9510 & 5.8996 & 6.5190 & 7.0311 & 7.7516 \\
\hline 0.7 & 2.1118 & 3.4722 & 4.0136 & 4.6635 & 5.5936 & 6.3151 & 6.9572 & 7.6767 \\
\hline 0.8 & 1.6820 & 2.5595 & 3.7287 & 4.4402 & 5.3514 & 5.6290 & 6.4634 & 6.9789 \\
\hline 0.9 & 1.0418 & 1.6253 & 2.4139 & 3.8312 & 4.0584 & 4.5263 & 4.9883 & 5.3557 \\
\hline 0.95 & 0.58091 & 1.2646 & 1.3811 & 2.3232 & 2.4816 & 2.9481 & 3.9027 & 4.3298 \\
\hline 0.99 & 0.13659 & 0.29412 & 0.62571 & 0.82832 & 1.1495 & 1.1668 & 1.3005 & 1.3755 \\
\hline \multicolumn{9}{|c|}{ SS mode } \\
\hline 0.6 & 1.9748 & 2.2247 & 3.4583 & 3.8137 & 4.6887 & 5.1173 & 6.3689 & 6.8035 \\
\hline 0.7 & 1.5025 & 2.0203 & 3.1470 & 3.7500 & 4.8337 & 4.9232 & 5.7668 & 6.2829 \\
\hline 0.8 & 1.0351 & 1.6964 & 2.7072 & 3.5994 & 4.3628 & 4.7714 & 5.1446 & 5.4338 \\
\hline 0.9 & 0.58640 & 1.1727 & 1.8904 & 2.6949 & 3.4066 & 3.5548 & 3.9428 & 4.7161 \\
\hline 0.95 & 0.33385 & 0.73534 & 1.4092 & 1.8017 & 2.0975 & 2.3467 & 3.3226 & 3.3259 \\
\hline 0.99 & 0.077925 & 0.17410 & 0.45756 & 0.71709 & 1.0539 & 1.1297 & 1.2520 & 1.3515 \\
\hline
\end{tabular}


Table 11 The first eight dimensionless frequencies $\Delta r_{1} \sqrt{\rho / \sqrt{G}}$ of various mode classifications for a $C F C F$ saddle-shaped shell panel with the size parameters: $R / r_{1}=1.5, \varphi_{0}=90^{\circ}, \theta_{0}=135^{\circ}, \theta_{1}=90^{\circ}$

\begin{tabular}{|c|c|c|c|c|c|c|c|c|}
\hline$r_{0} / r_{1}$ & $\Omega_{1}$ & $\Omega_{2}$ & $\Omega_{3}$ & $\Omega_{4}$ & $\Omega_{5}$ & $\Omega_{6}$ & $\Omega_{7}$ & $\Omega_{8}$ \\
\hline \multicolumn{9}{|c|}{ AA mode } \\
\hline 0.6 & 3.2992 & 4.6710 & 5.0488 & 6.3832 & 7.7728 & 8.2371 & 8.6840 & 9.2223 \\
\hline 0.7 & 3.1942 & 4.6229 & 5.1826 & 5.9622 & 7.6763 & 8.0878 & 8.8582 & 9.4913 \\
\hline 0.8 & 2.8764 & 4.4294 & 5.1499 & 5.7068 & 7.1703 & 7.7380 & 8.3512 & 8.9620 \\
\hline 0.9 & 2.0317 & 3.4158 & 4.8696 & 5.5296 & 5.6544 & 5.8106 & 7.6662 & 8.0917 \\
\hline 0.95 & 1.3076 & 2.2105 & 3.3576 & 3.5157 & 4.9008 & 5.3675 & 5.3953 & 5.9008 \\
\hline 0.99 & 0.74408 & 0.89355 & 1.2044 & 1.3567 & 1.6548 & 1.7427 & 1.8711 & 2.0050 \\
\hline \multicolumn{9}{|c|}{ AS mode } \\
\hline 0.6 & 3.2890 & 4.0962 & 4.7566 & 6.1631 & 6.9826 & 7.8304 & 8.2080 & 8.7367 \\
\hline 0.7 & 3.2491 & 3.9748 & 4.8578 & 6.0960 & 6.8781 & 7.6200 & 7.7443 & 9.1164 \\
\hline 0.8 & 2.9346 & 3.8162 & 4.9066 & 5.9866 & 6.3082 & 7.1382 & 7.3068 & 9.2102 \\
\hline 0.9 & 2.0639 & 3.1170 & 4.4086 & 4.8698 & 5.5224 & 5.9433 & 7.0564 & 7.2591 \\
\hline 0.95 & 1.3314 & 2.1472 & 2.8199 & 3.3584 & 4.3748 & 4.8791 & 5.3789 & 5.8428 \\
\hline 0.99 & 0.74313 & 0.93633 & 1.2141 & 1.3711 & 1.5659 & 1.6719 & 1.7420 & 2.0500 \\
\hline \multicolumn{9}{|c|}{ SA mode } \\
\hline 0.6 & 2.0818 & 2.8962 & 5.1166 & 5.6595 & 6.8371 & 7.5284 & 8.1710 & 8.4114 \\
\hline 0.7 & 2.0429 & 2.9552 & 4.4555 & 5.5043 & 6.8719 & 7.8302 & 8.2509 & 8.2821 \\
\hline 0.8 & 1.9023 & 3.0573 & 3.6546 & 5.0193 & 6.7511 & 7.1207 & 7.7614 & 8.1593 \\
\hline 0.9 & 1.6303 & 2.6952 & 3.2082 & 3.6575 & 4.7000 & 5.5606 & 7.3423 & 7.3859 \\
\hline 0.95 & 1.4301 & 2.1586 & 2.3377 & 3.1270 & 3.2725 & 3.7237 & 4.7351 & 4.7936 \\
\hline 0.99 & 0.73328 & 1.0804 & 1.2473 & 1.3615 & 1.5608 & 1.7969 & 1.8489 & 2.0940 \\
\hline \multicolumn{9}{|c|}{ SS mode } \\
\hline 0.6 & 2.5222 & 3.2717 & 4.7513 & 5.7488 & 6.5440 & 7.2741 & 7.7570 & 8.1678 \\
\hline 0.7 & 2.3699 & 3.0422 & 4.6670 & 5.5358 & 6.4735 & 6.7492 & 7.9378 & 8.3116 \\
\hline 0.8 & 2.0832 & 2.8734 & 4.5600 & 5.0206 & 5.2462 & 6.7296 & 7.9142 & 8.3634 \\
\hline 0.9 & 1.7007 & 2.5930 & 3.5264 & 3.6757 & 4.4564 & 5.5553 & 6.1408 & 6.5221 \\
\hline 0.95 & 1.4663 & 2.2179 & 2.3785 & 2.6775 & 3.6666 & 3.8978 & 4.3997 & 4.4492 \\
\hline 0.99 & 0.74424 & 1.0341 & 1.2564 & 1.5276 & 1.5465 & 1.5949 & 1.8213 & 2.0967 \\
\hline
\end{tabular}




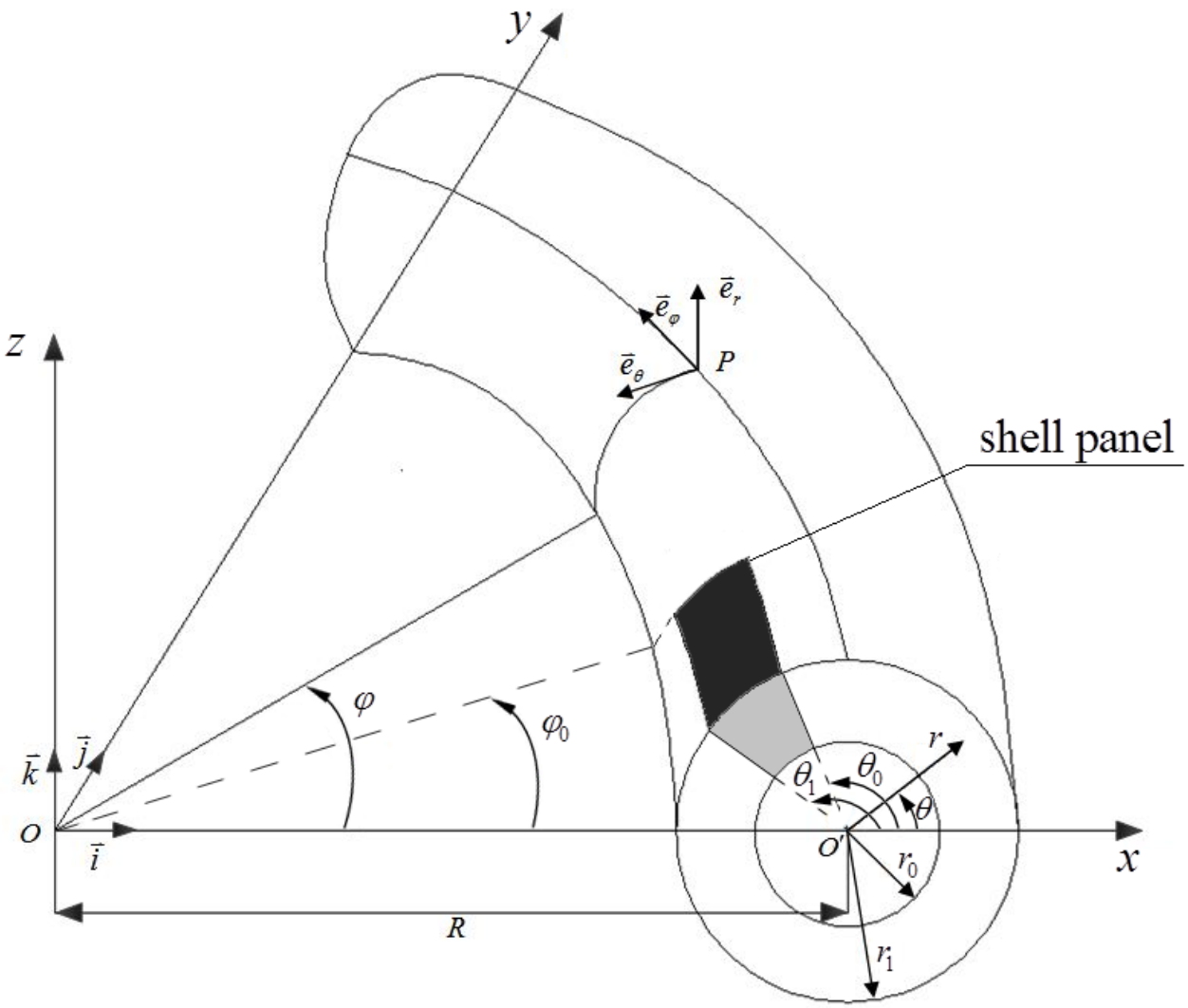

Figure 1 The shell panel from a hollow ring torus with annular cross-section as well as its coordinate system and sizes. 


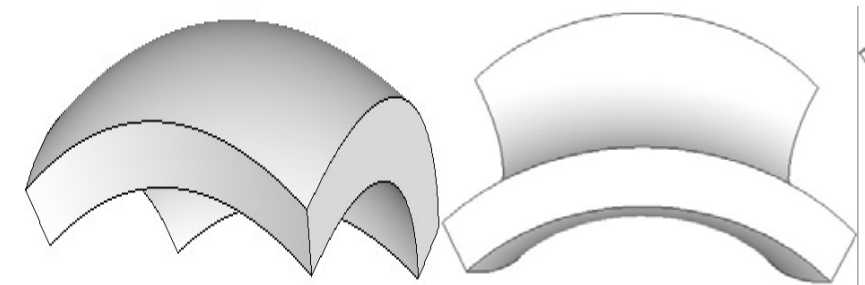

(a) Cap-shaped panel (b) Saddle-shaped panel

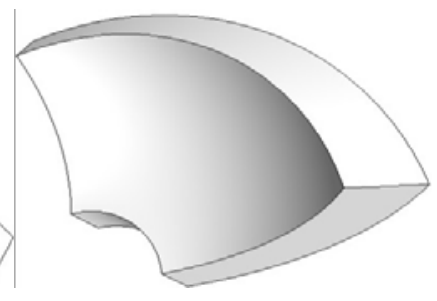

(c) Sectorial-shaped panel

Figure 2 Three typical doubly-curved shell panels. 


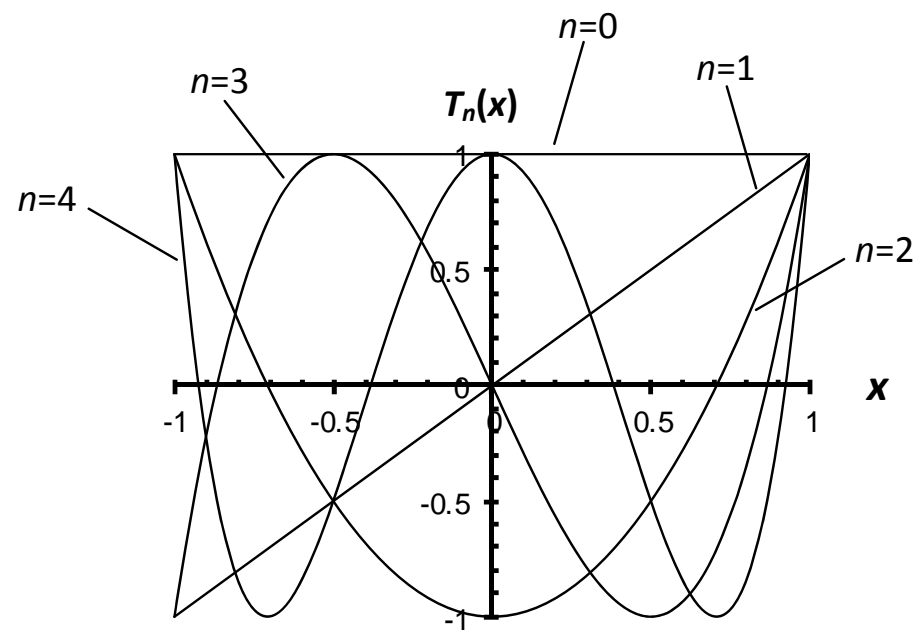

Figure 3 The first five terms of Chebyshev polynomials $T_{n}(x) \quad(n=1,2,3,4,5)$. 


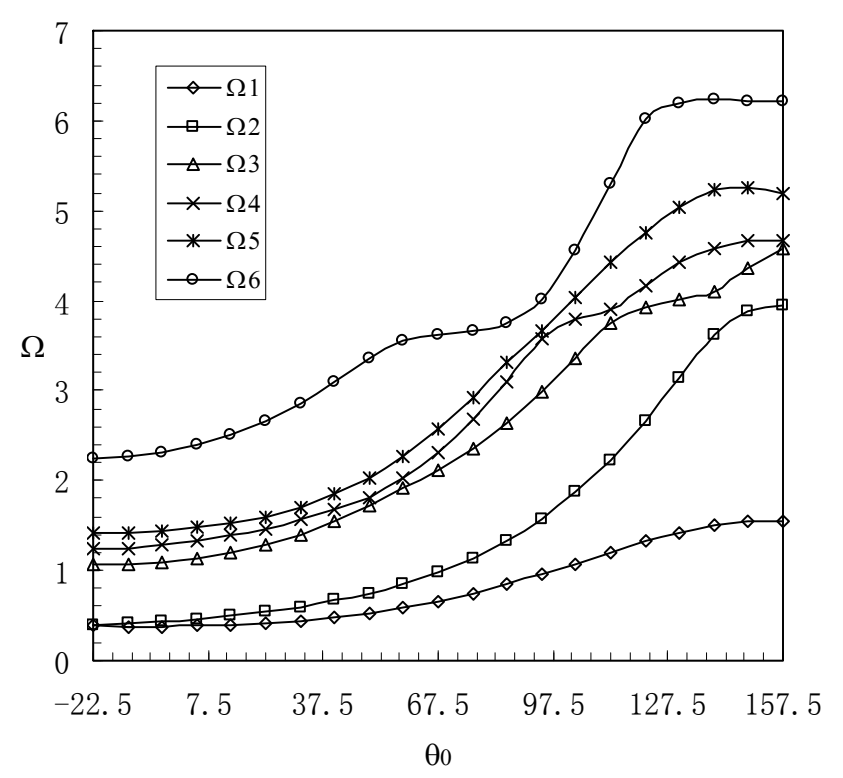

(a) Antisymmetric modes

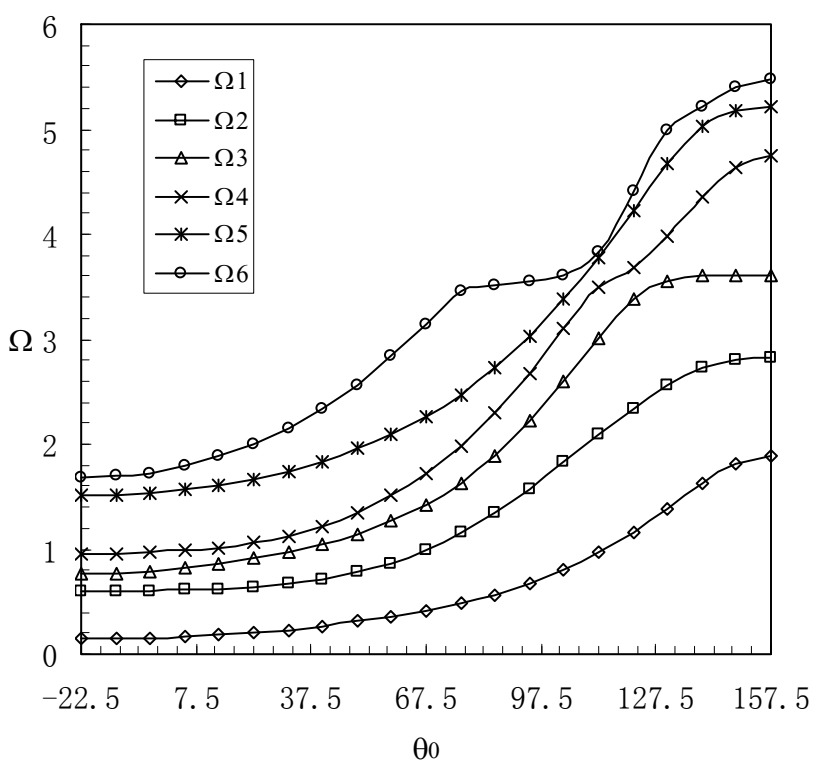

(b) Symmetric modes

Figure 4 The first six non-zero dimensionless frequencies of antisymmetric and symmetric modes in the toroidal direction for FFFF shell panels with the size parameters: $R / r_{1}=1.5, r_{0} / r_{1}=0.8, \theta_{1}=45^{\circ}, p_{0}=90^{\circ}$. 


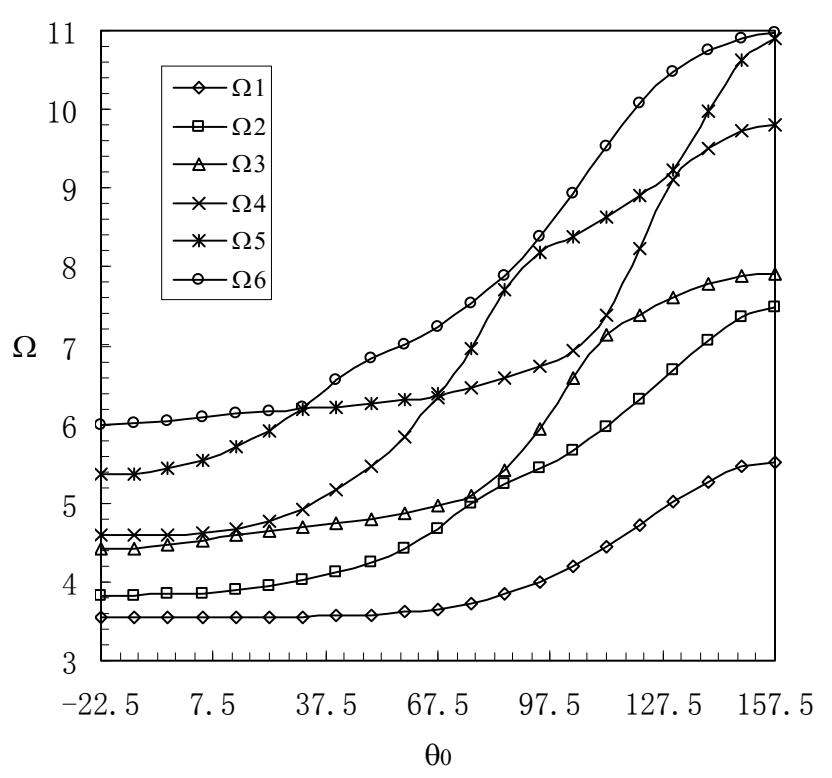

(a) Antisymmetric modes

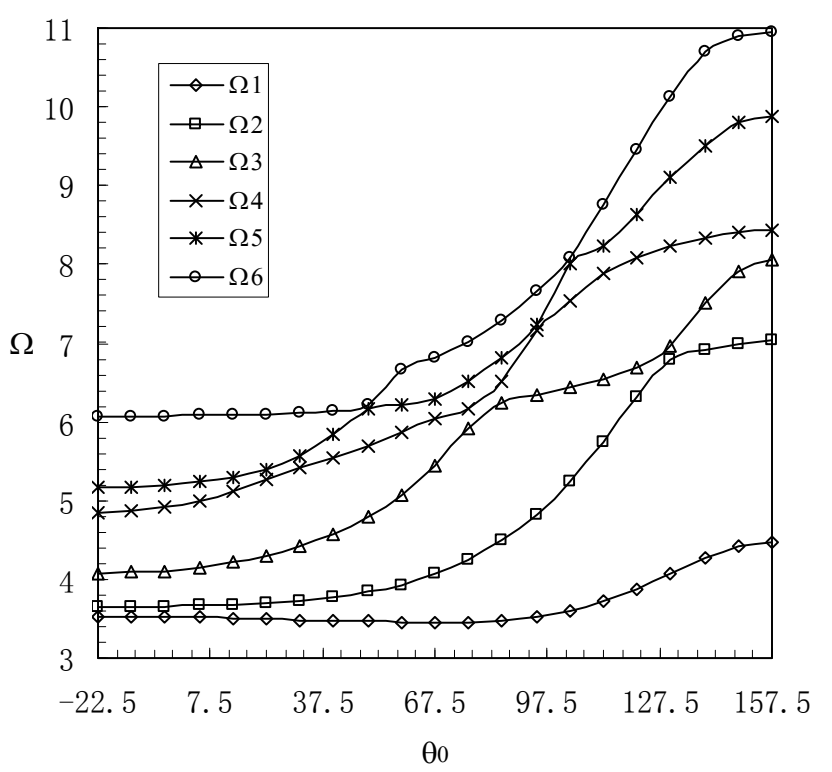

(b) Symmetric modes

Figure 5 The first six dimensionless frequencies of antisymmetric and symmetric modes in the toroidal direction for $C C C C$ shell panels with the size parameters: $R / r_{1}=1.5, r_{0} / r_{1}=0.8, \theta_{1}=45^{\circ}, \varphi_{0}=90^{\circ}$. 


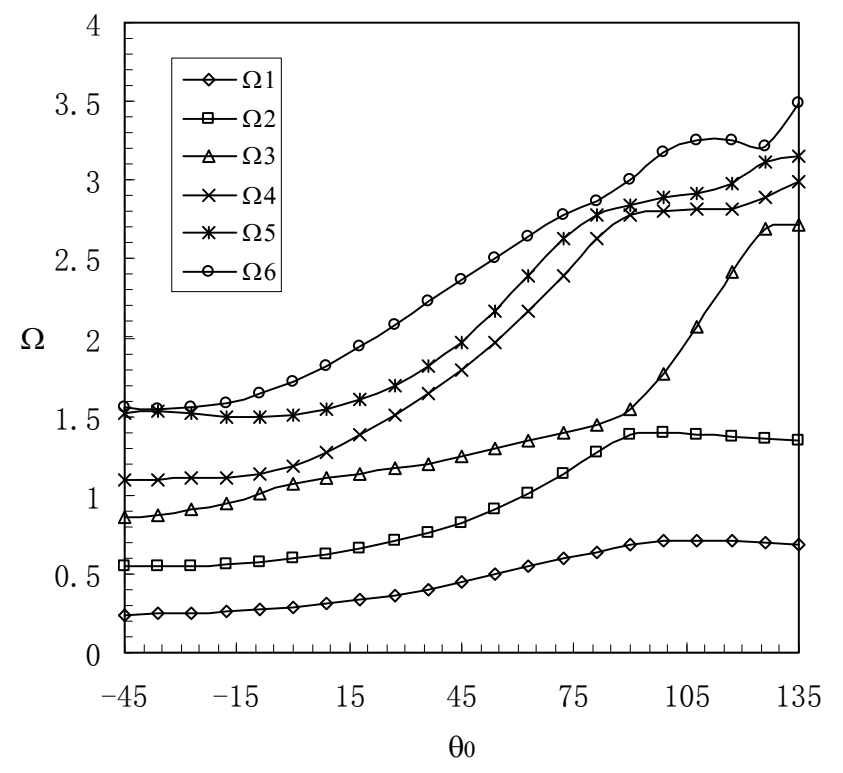

(a) Antisymmetric modes

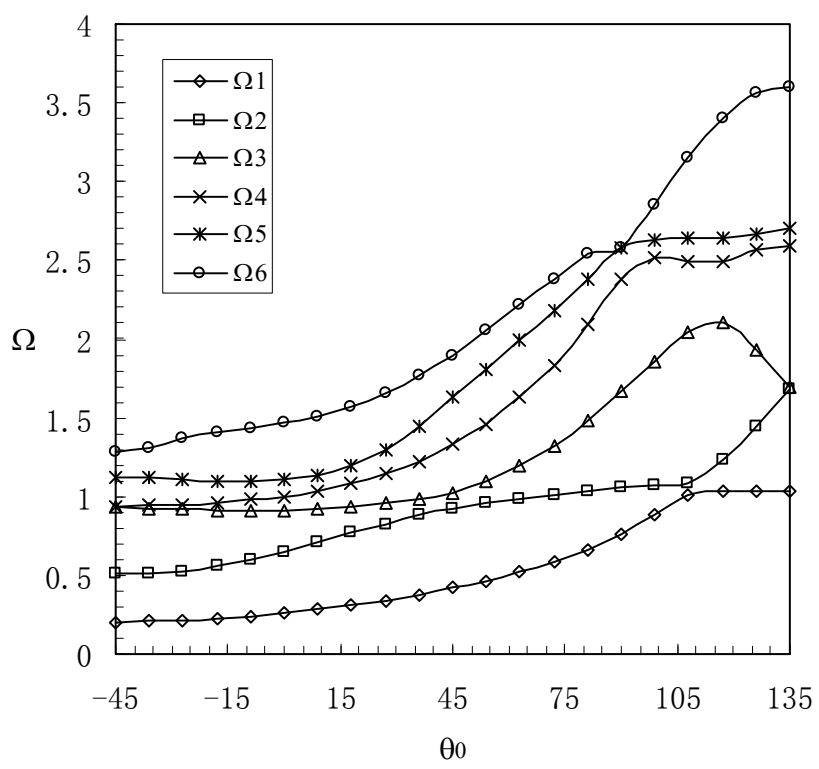

(b) Symmetric modes

Figure 6 The first six non-zero dimensionless frequencies of antisymmetric and symmetric modes in the toroidal direction for FFFF shell panels with the size parameters: $R / r_{1}=1.5, r_{0} / r_{1}=0.8, \theta_{1}=90^{\circ}, p_{0}=90^{\circ}$. 


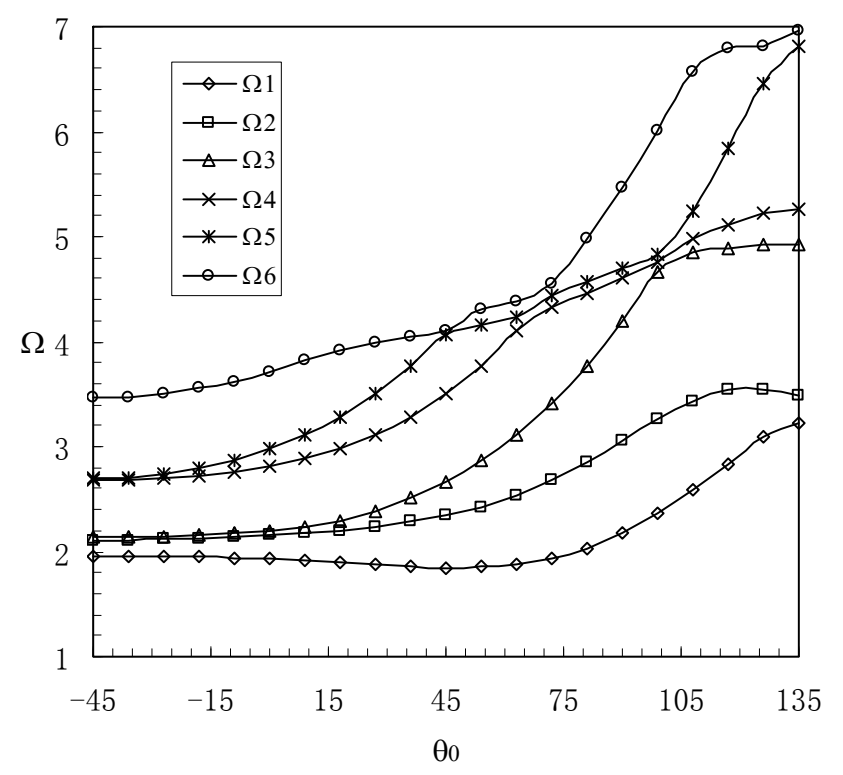

(c) Antisymmetric modes

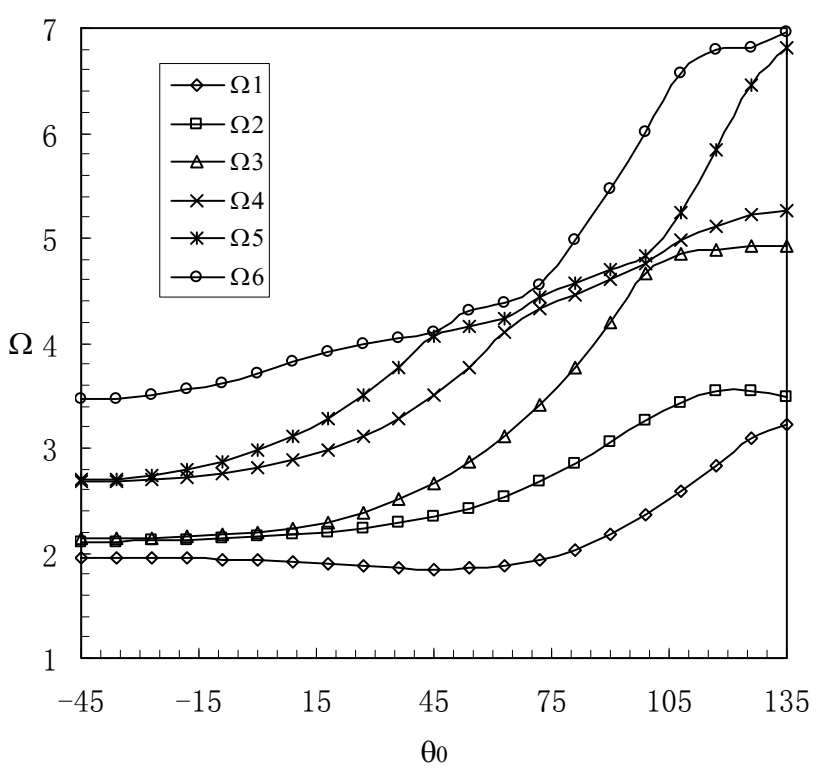

(d) Symmetric modes

Figure 7 The first six dimensionless frequencies of antisymmetric and symmetric modes in the toroidal direction for $C C C C$ shell panels with the size parameters: $R / r_{1}=1.5, r_{0} / r_{1}=0.8, \theta_{1}=90^{\circ}, \varphi_{0}=90^{\circ}$. 


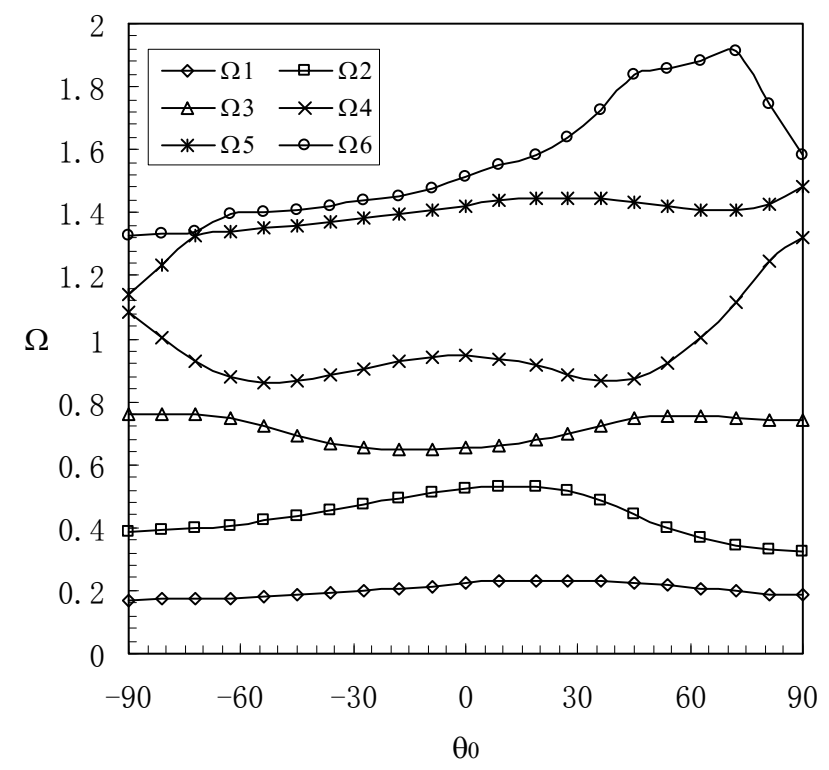

(a) Antisymmetric modes

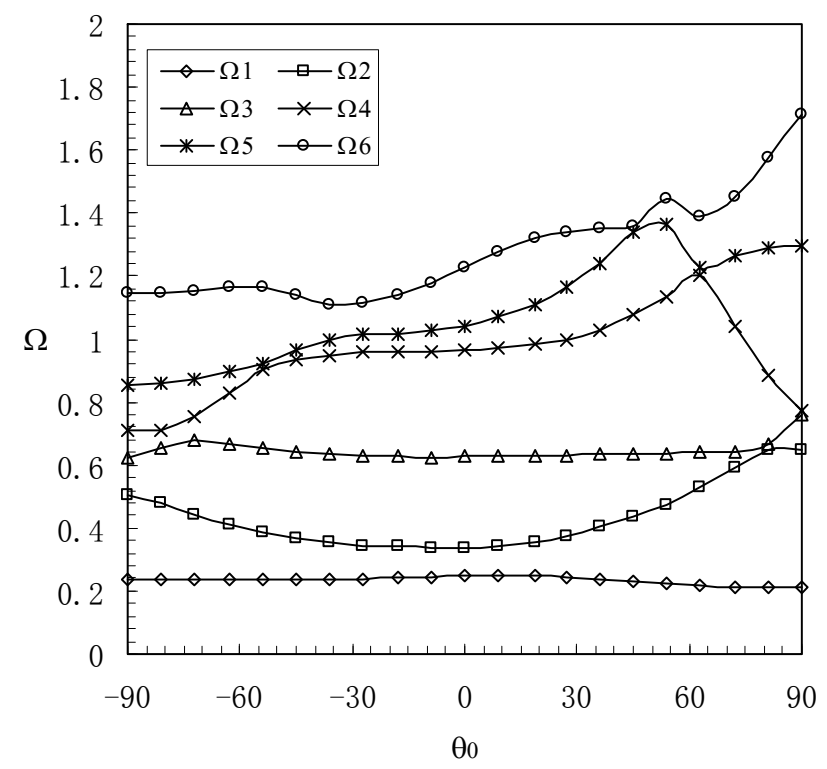

(b) Symmetric modes

Figure 8 The first six non-zero dimensionless frequencies of antisymmetric and symmetric modes in the toroidal direction for FFFF shell panels with the size parameters: $R / r_{1}=1.5, r_{0} / r_{1}=0.8, \theta_{1}=180^{\circ}, \varphi_{0}=90^{\circ}$. 


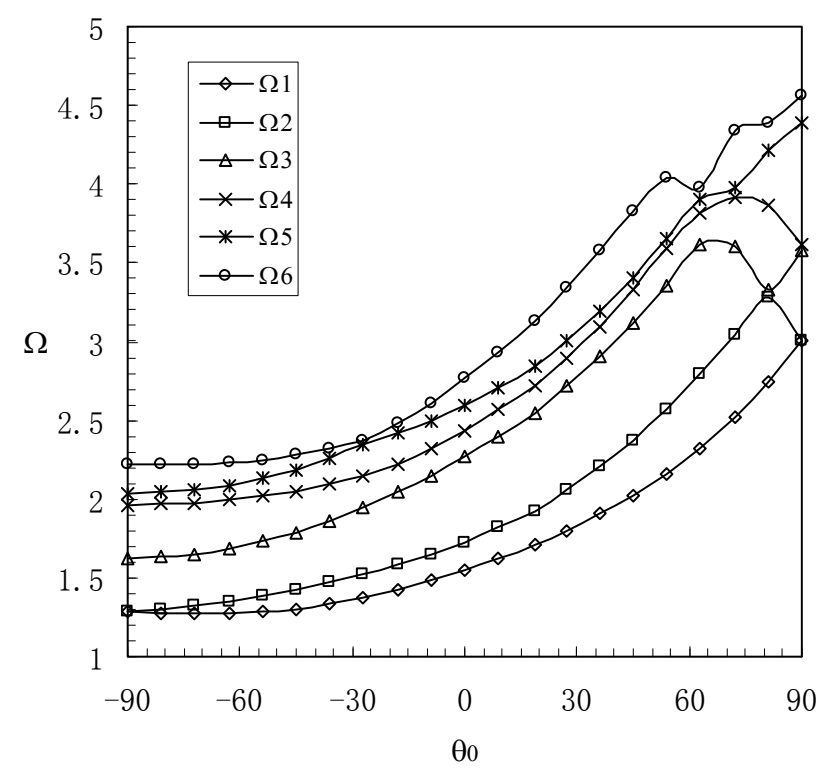

(a) Antisymmetric modes

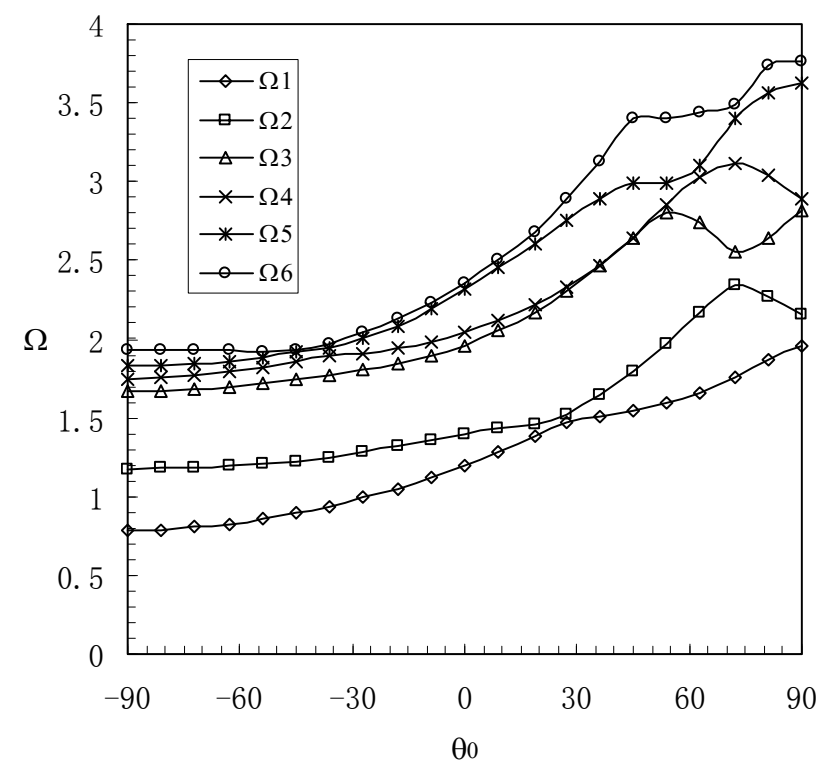

(b) Symmetric modes

Figure 9 The first six dimensionless frequencies of antisymmetric and symmetric modes in the toroidal direction for $C C C C$ shell panels with the size parameters: $R / r_{1}=1.5, r_{0} / r_{1}=0.8, \theta_{1}=180^{\circ}, \varphi_{0}=90^{\circ}$ 


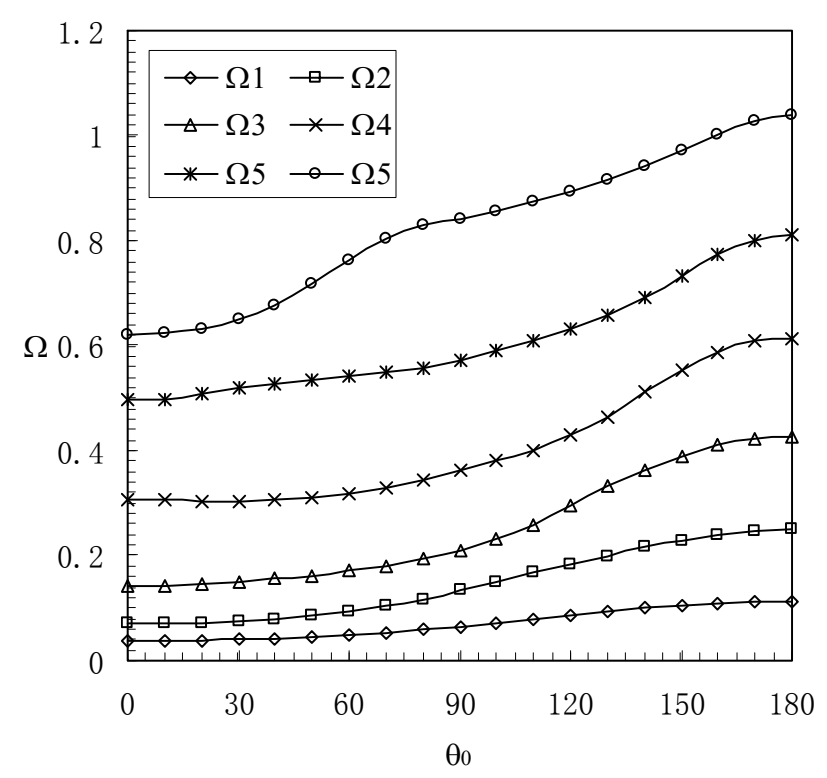

(a) Antisymmetric modes

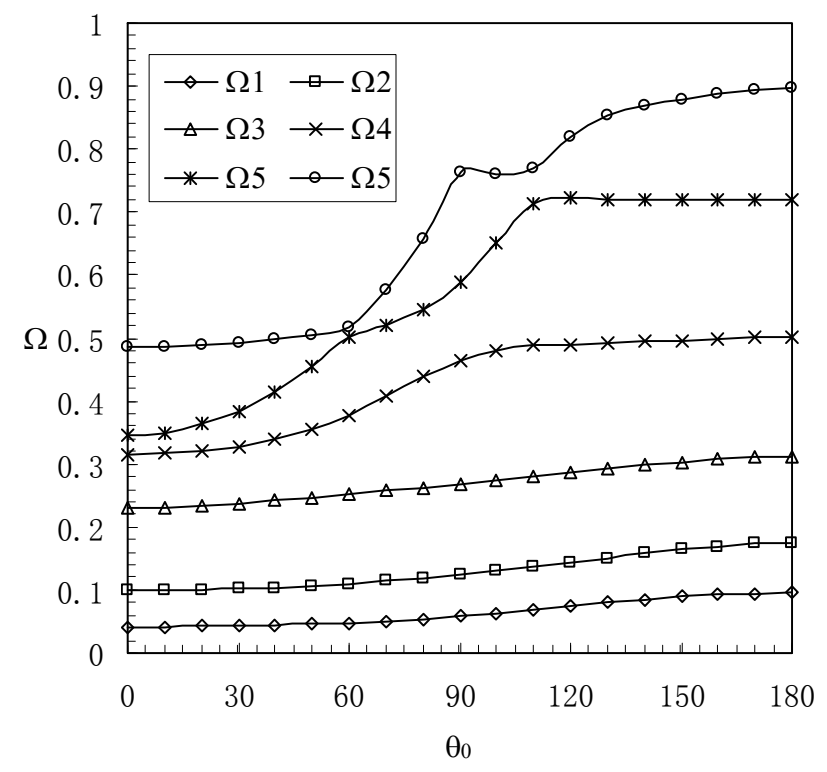

(b) Symmetric modes

Figure 10 The first six non-zero dimensionless frequencies of antisymmetric and symmetric modes in the toroidal direction for $F F F F$ shell panels with the size parameters: $R / r_{1}=1.5, r_{0} / r_{1}=0.8, \theta_{1}=360^{\circ}, \varphi_{0}=90^{\circ}$. 


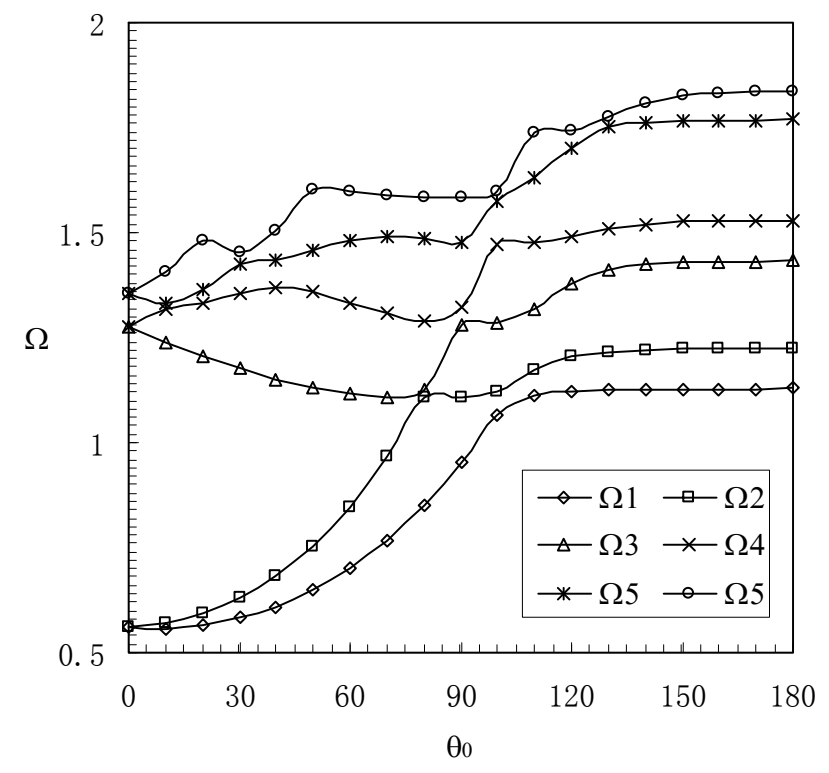

(a) Antisymmetric modes

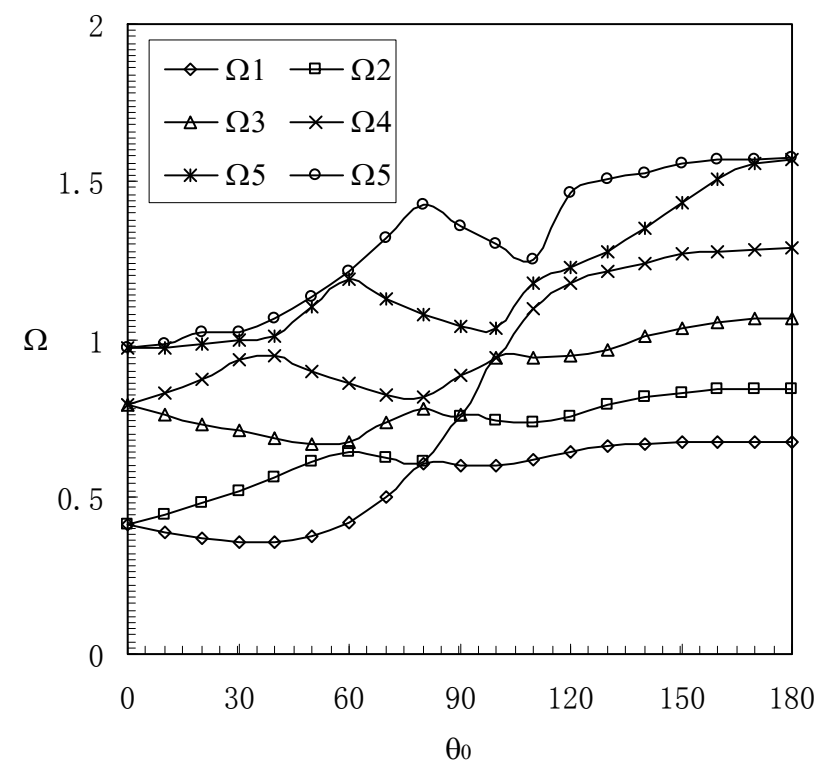

(b) Symmetric modes

Figure 11 The first six dimensionless frequencies of antisymmetric and symmetric modes in the toroidal direction for $C F C F$ shell panels with the size parameters: $R / r_{1}=1.5, r_{0} / r_{1}=0.8, \theta_{1}=360^{\circ}, \varphi_{0}=90^{\circ}$. 


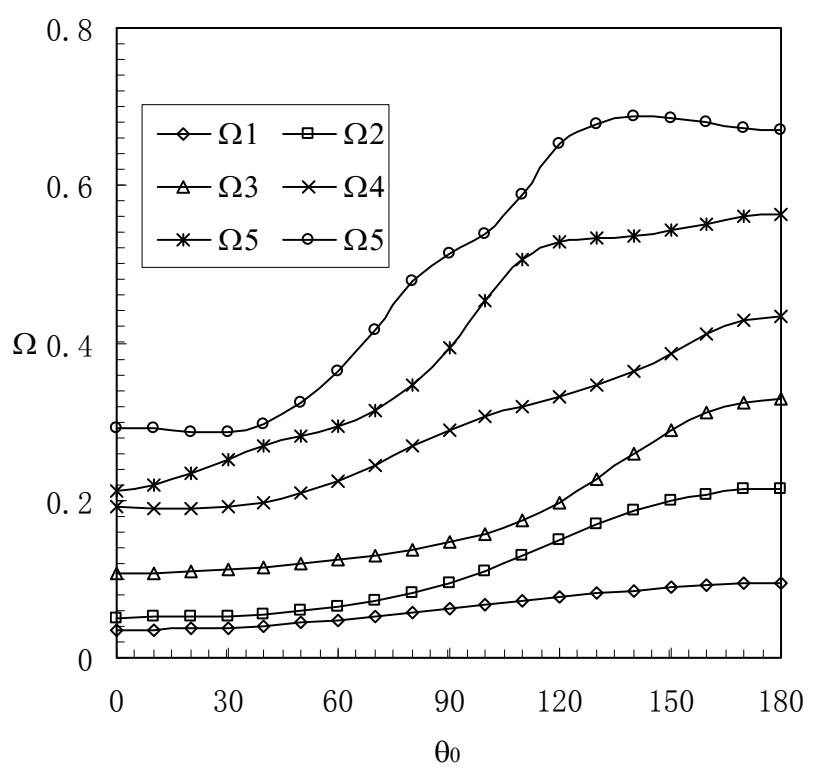

(a) Antisymmetric modes

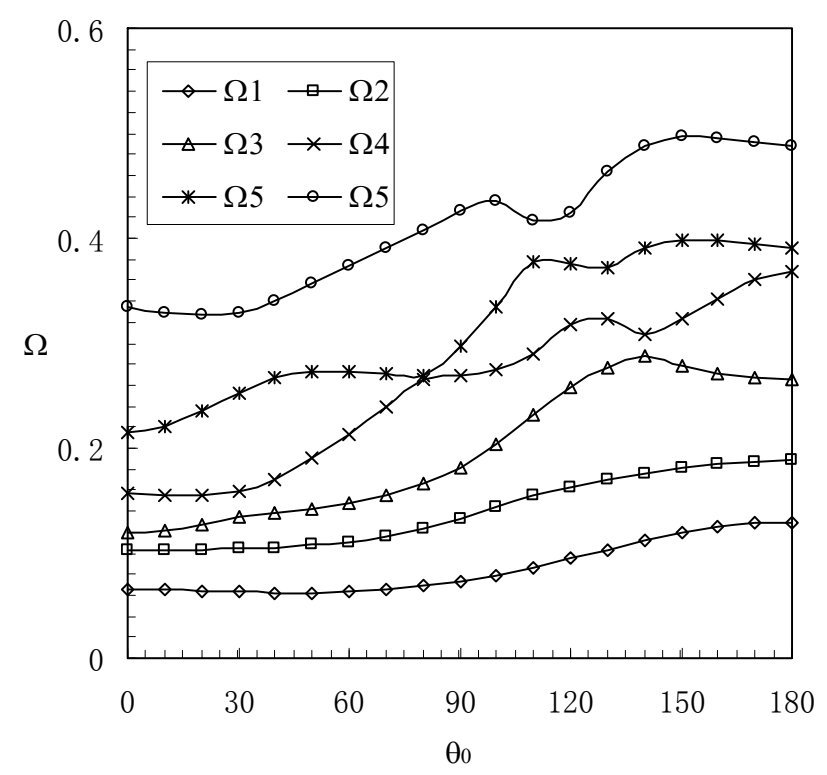

(b) Symmetric modes

Figure 12 The first six non-zero dimensionless frequencies of antisymmetric and symmetric modes in the toroidal direction for FFFF shell panels with the size parameters: $R / r_{1}=1.5, r_{0} / r_{1}=0.8, \theta_{1}=360^{\circ}, \varphi_{0}=180^{\circ}$. 


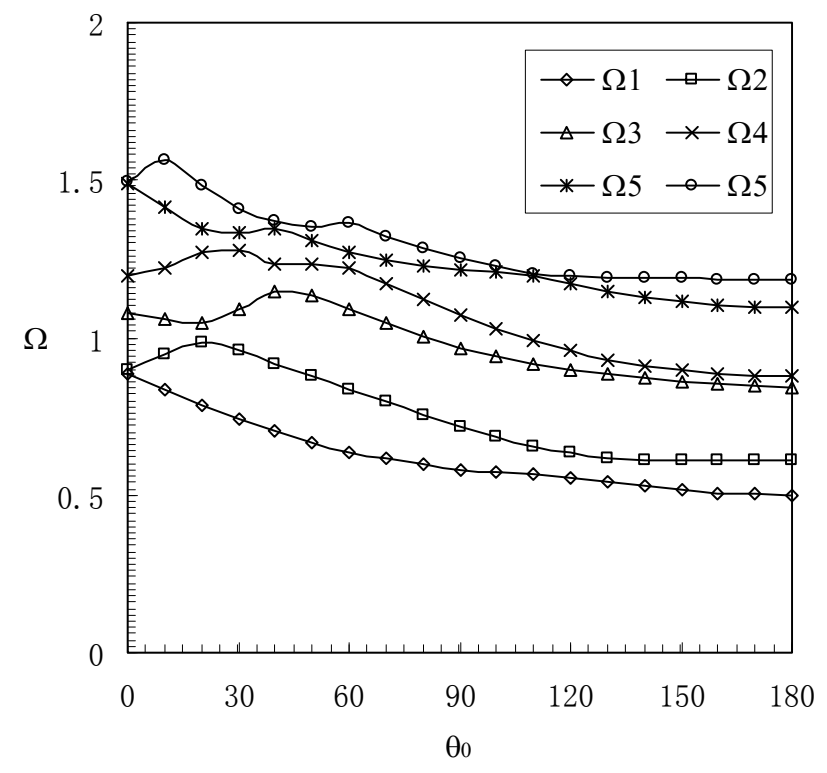

(a) Antisymmetric modes

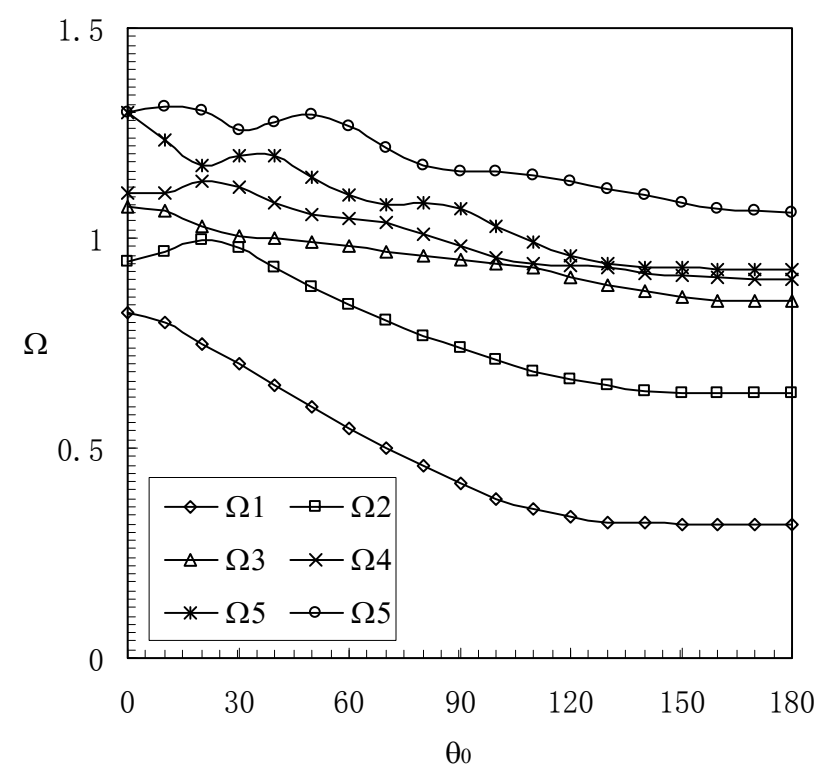

(b) Symmetric modes

Figure 13 The first six dimensionless frequencies of antisymmetric and symmetric modes in the toroidal direction for $C F C F$ shell panels with the size parameters: $R / r_{1}=1.5, r_{0} / r_{1}=0.8, \theta_{1}=360^{6}, \varphi_{0}=180^{\circ}$. 


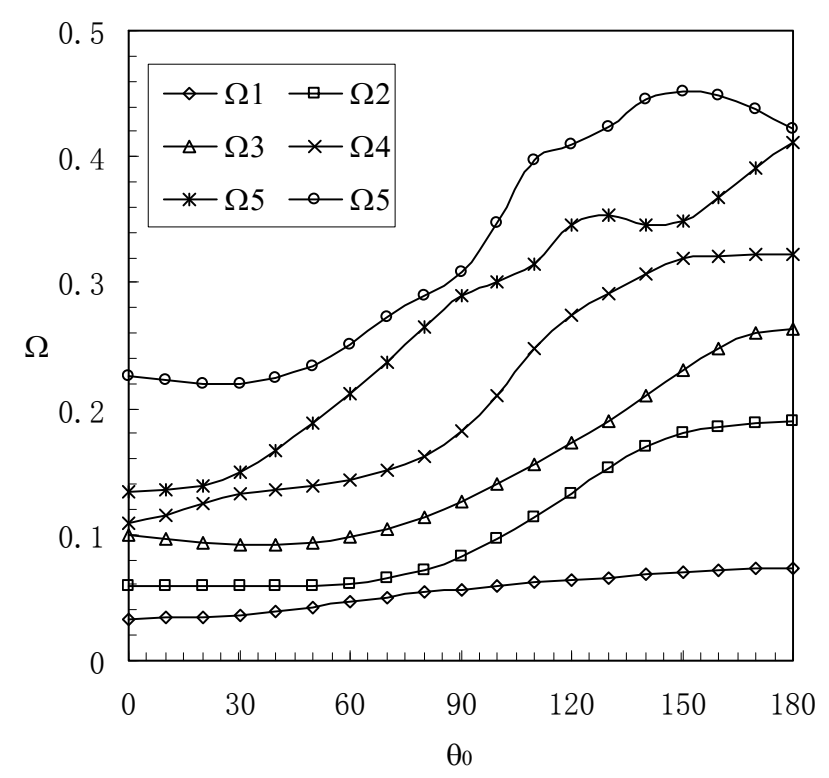

(a) Antisymmetric modes

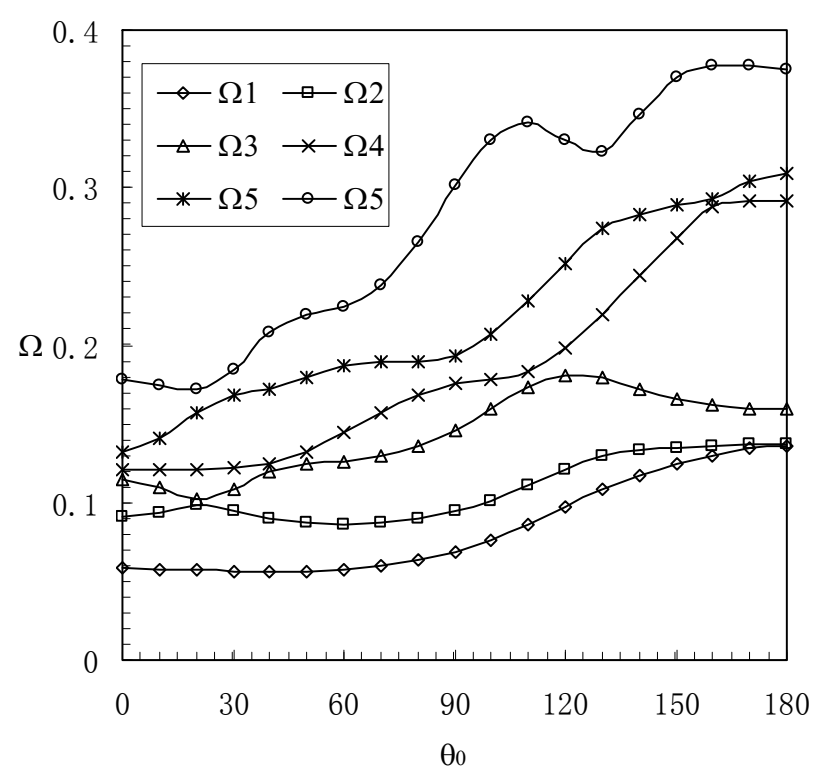

(b) Symmetric modes

Figure 14 The first six non-zero dimensionless frequencies of antisymmetric and symmetric modes in the toroidal direction for FFFF shell panels with the size parameters: $R / r_{1}=1.5, r_{0} / r_{1}=0.8, \theta_{1}=360^{\circ}, \varphi_{0}=270^{\circ}$. 


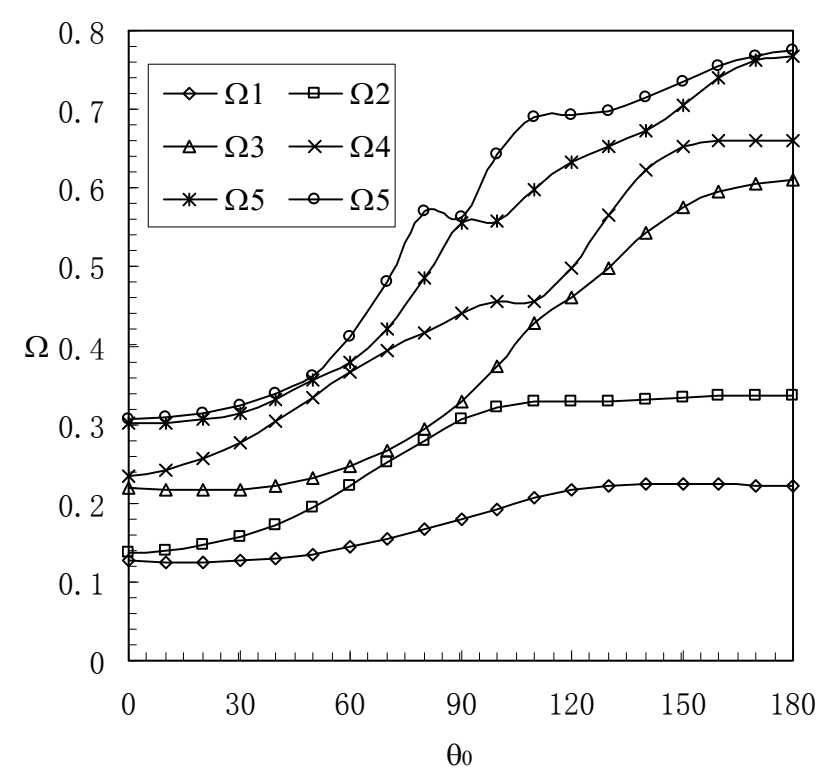

(a) Antisymmetric modes

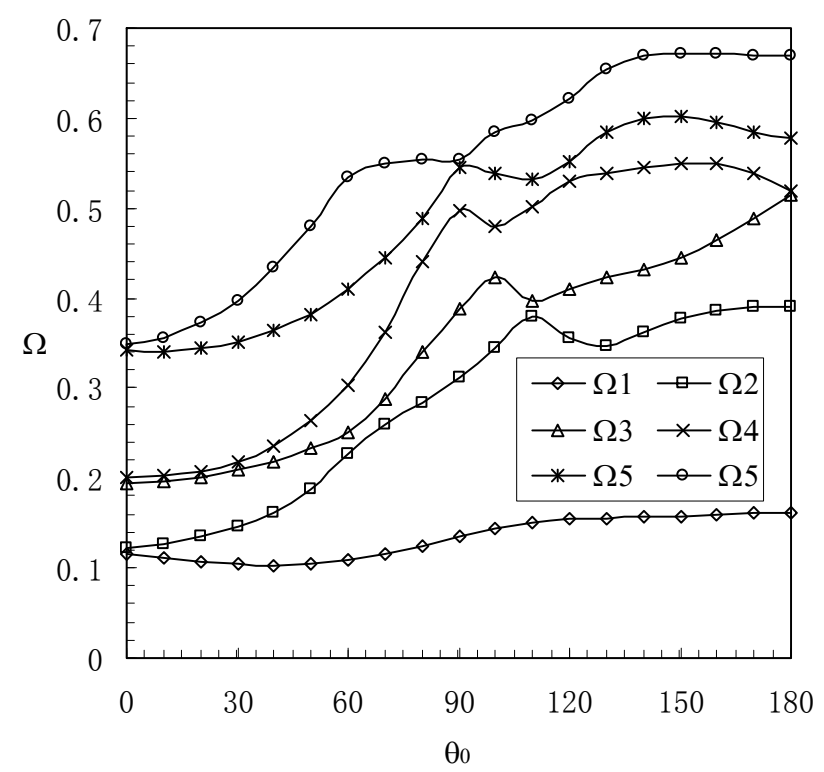

(b) Symmetric modes

Figure 15 The first six dimensionless frequencies of antisymmetric and symmetric modes in the toroidal direction for $C F C F$ shell panels with the size parameters: $R / r_{1}=1.5, r_{0} / r_{1}=0.8, \theta_{1}=360^{\circ}, \varphi_{0}=270^{\circ}$. 


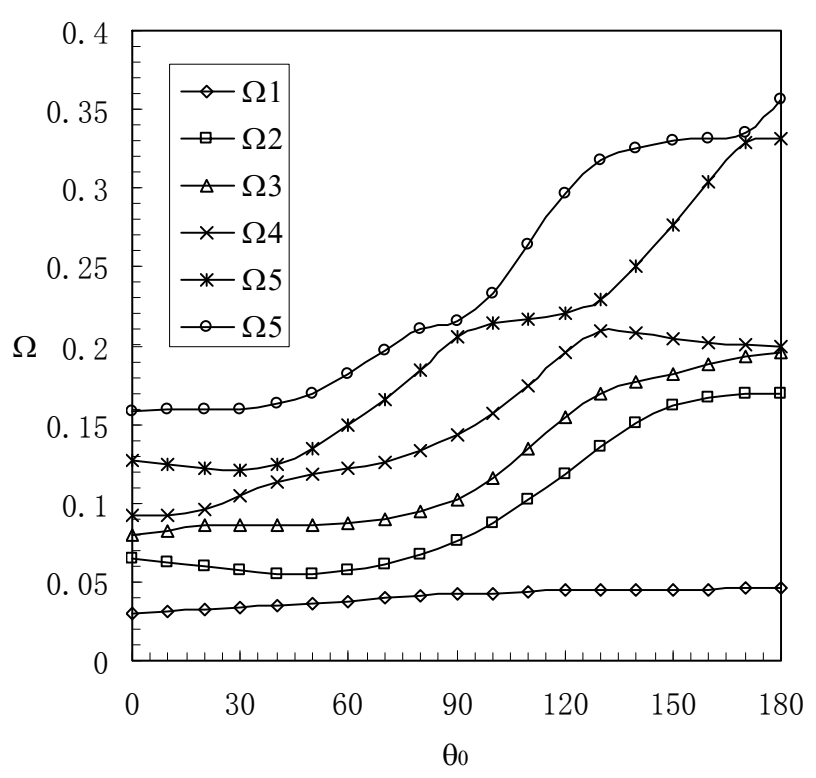

(a) Antisymmetric modes

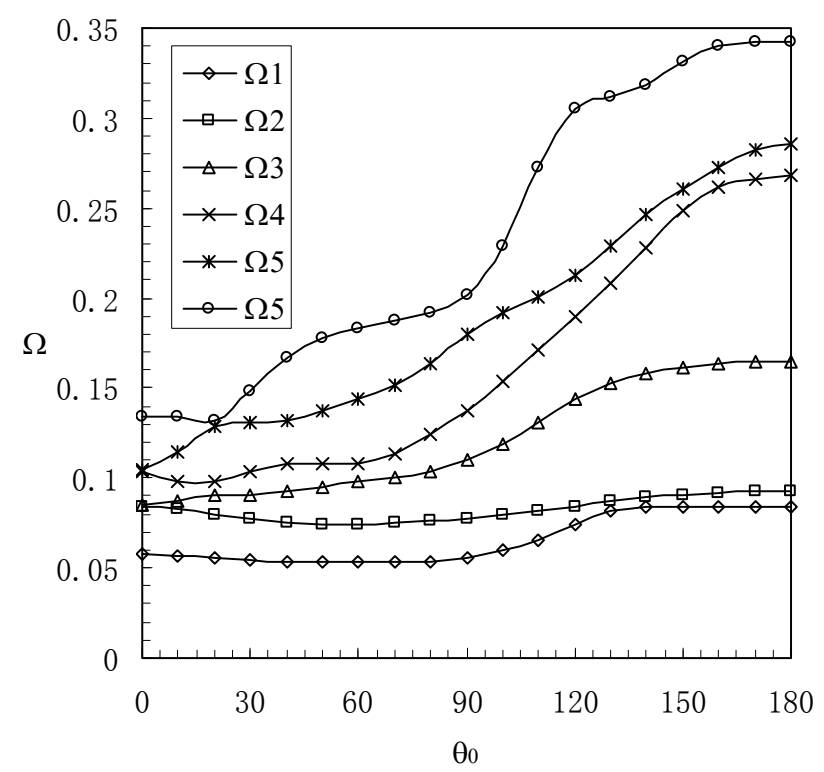

(b) Symmetric modes

Figure 16 The first six non-zero dimensionless frequencies of antisymmetric and symmetric modes in the toroidal direction for FFFF shell panels with the size parameters: $R / r_{1}=1.5, r_{0} / r_{1}=0.8, \theta_{1}=360^{\circ}, \varphi_{0}=\$ 60^{\circ}$. 


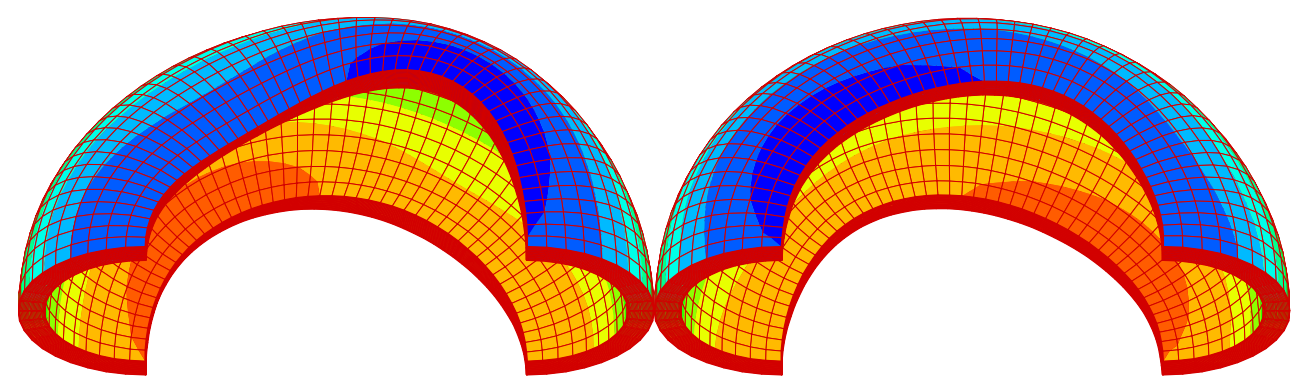

(a) First AA mode, $\Omega_{1}=0.51231$

(b) Second AA mode, $\Omega_{2}=0.57738$

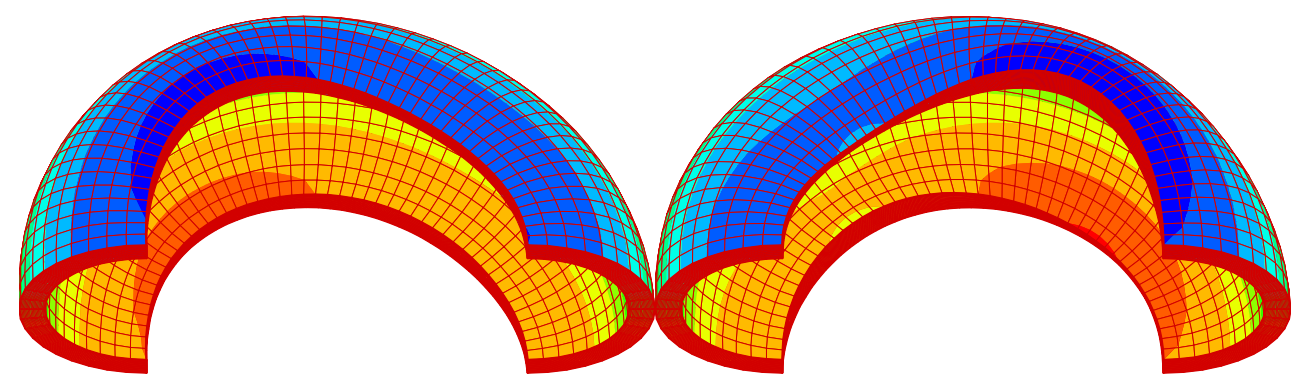

(c) First AS mode, $\Omega_{1}=0.28927$

(d) Second AS mode, $\Omega_{2}=0.61332$

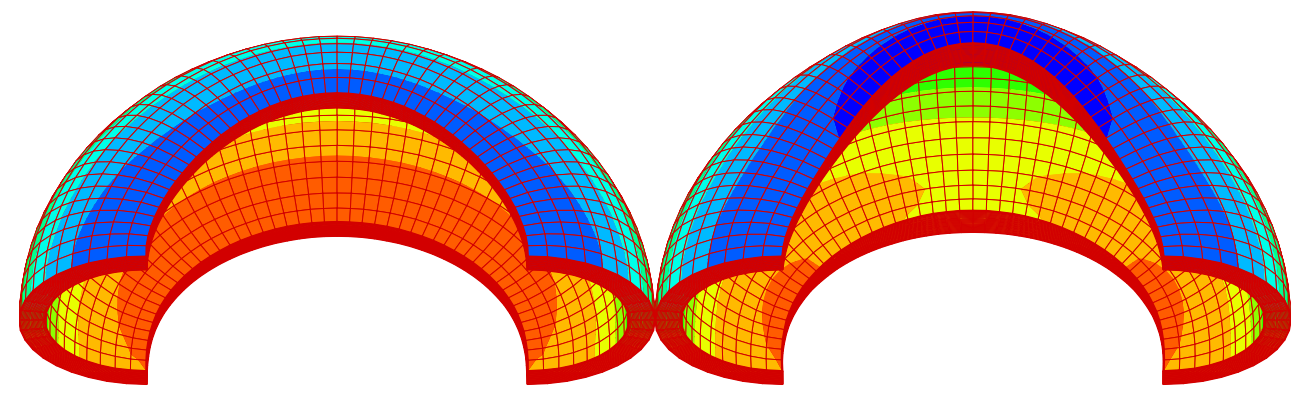

(e) First SA mode, $\Omega_{1}=0.25156$

(f) Second AS mode, $\Omega_{2}=0.52713$ 


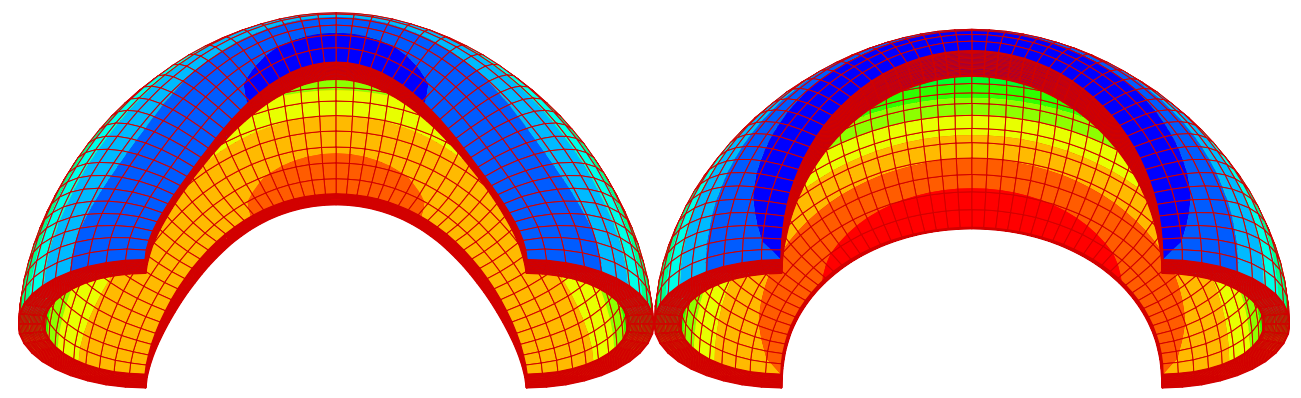

(g) First SS mode, $\Omega_{1}=0.48125$

(h) Second SS mode, $\Omega_{2}=0.49840$

Figure 17 The first two modes of various mode classifications of CFCF shell panel with the size parameters: $\quad R / r_{1}=1.5, \quad r_{0} / r_{1}=0.8, \quad \theta_{1}=180^{\circ}, \quad \sigma_{0}=\mathbf{- 1} 90^{\circ}$, $\varphi_{0}=180^{\circ}$ 


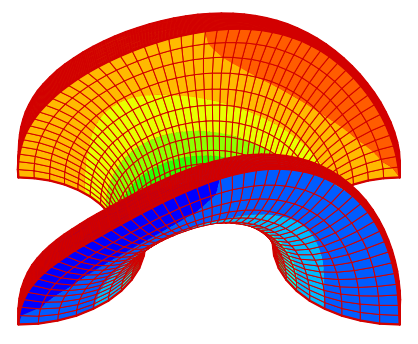

(a) First AA mode, $\Omega_{1}=0.49001$

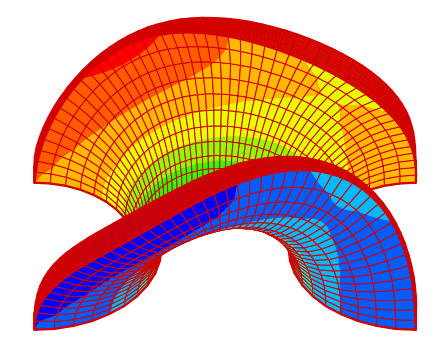

(c) First AS mode, $\Omega_{1}=0.52317$

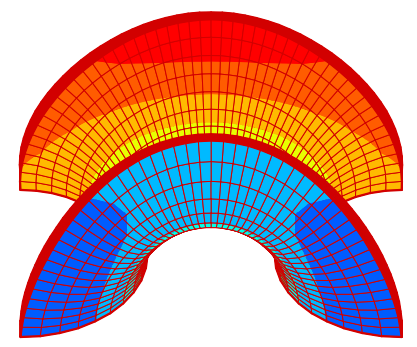

(e) First SA mode, $\Omega_{1}=0.49408$

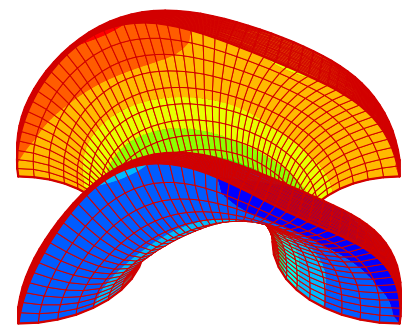

(b) Second AA mode, $\Omega_{2}=1.0030$

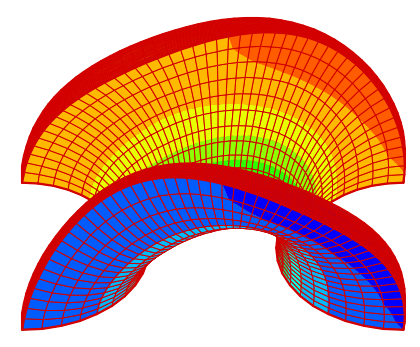

(d) Second AS mode, $\Omega_{2}=0.88040$

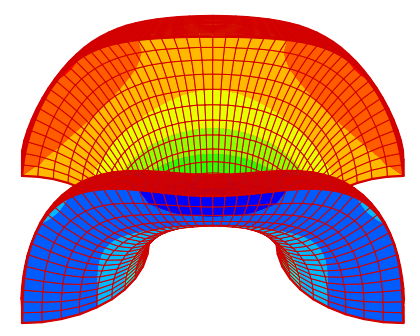

(f) Second SA mode, $\Omega_{2}=0.80461$ 


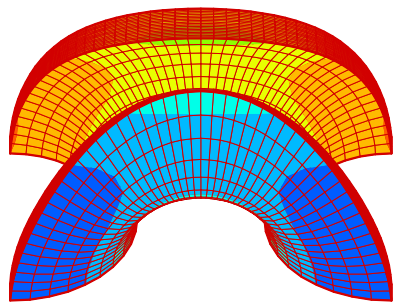

(g) First SS mode, $\Omega_{1}=0.54681$

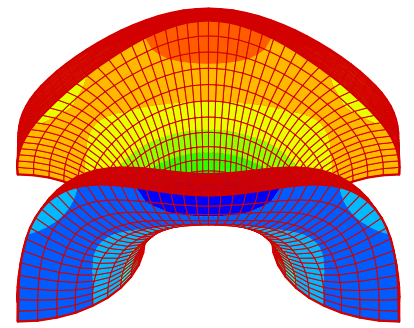

(h) Second SS mode, $\Omega_{2}=0.80782$

Figure 18 The first two modes of various mode classifications of CFCF shell panel with the size parameters: $R / r_{1}=1.5, r_{0} / r_{1}=0.8, \theta_{1}=180^{\circ}, \theta_{0}=90^{\circ}, \varphi_{0}=180^{\circ}$. 


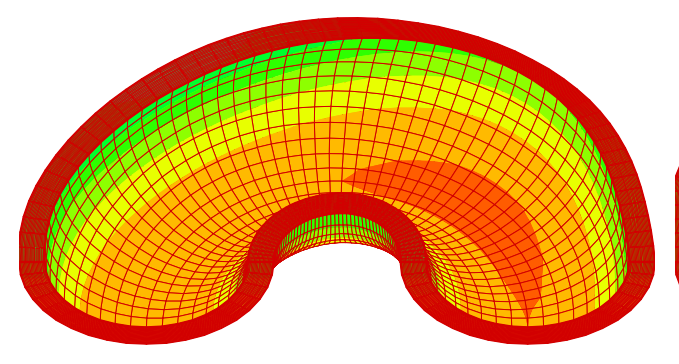

(a) First A mode, $\Omega_{1}=0.21581$

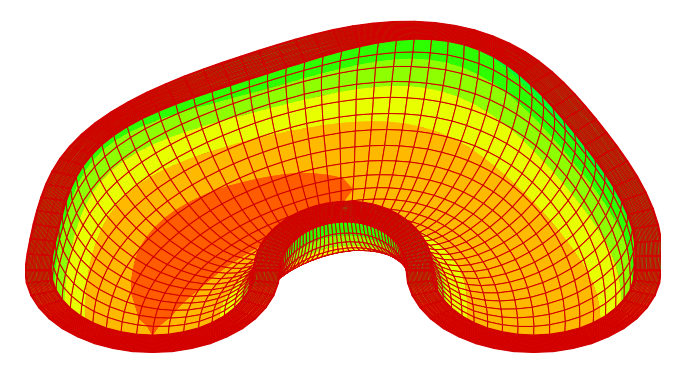

(c) Third A mode, $\Omega_{3}=0.56877$

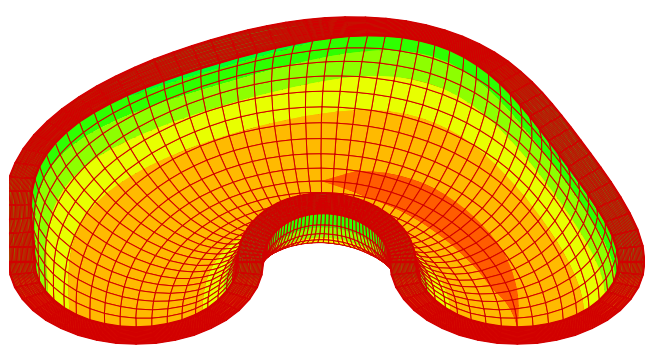

(b) Second A mode $\Omega_{2}=0.44956$

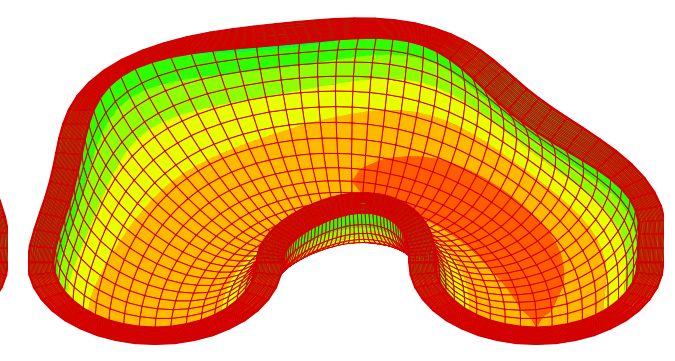

(d) Fourth A mode $\Omega_{4}=0.79684$

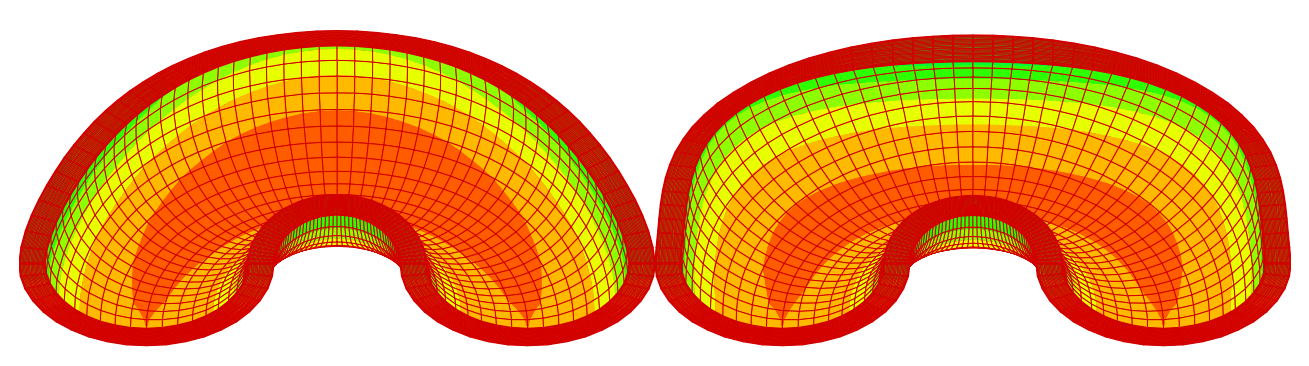

(e) First S mode, $\Omega_{1}=0.16076$

(f) Second S mode $\Omega_{2}=0.35009$ 


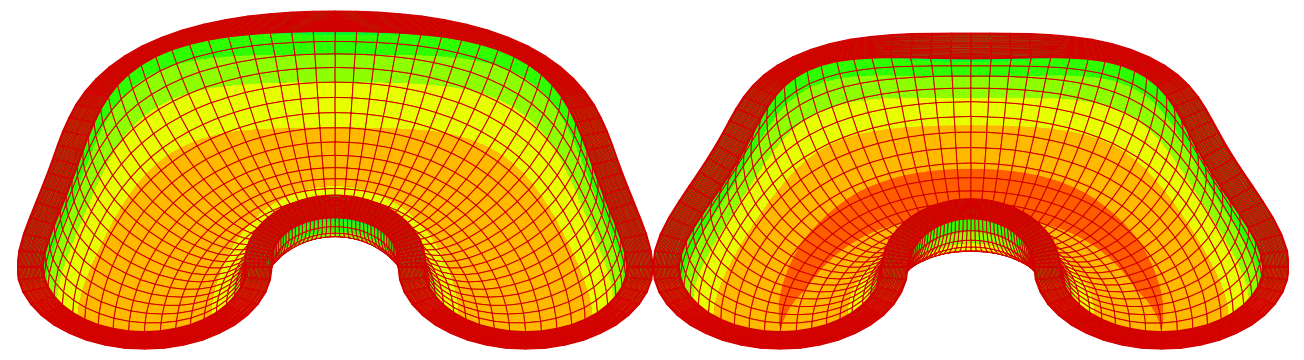

(g) Third S mode, $\Omega_{1}=0.57096$

(h) Fourth $\mathrm{S}$ mode $\Omega_{2}=0.67727$

Figure 19 The first two modes of various mode classifications of CFCF shell panel with the size parameters: $R / r_{1}=1.5, r_{0} / r_{1}=0.8, \theta_{1}=180^{\circ}, \theta_{0}=0^{\circ}, \varphi_{0}=180^{\circ}$. 